\title{
The Cherenkov Telescope Array transient and multi-messenger program
}

\section{Alessandro Carosi, ${ }^{a, *}$ Alicia López-Oramas ${ }^{b}$ and Francesco Longo ${ }^{c}$ on behalf of the CTA Collaboration}

(a complete list of authors can be found at the end of the proceedings)

${ }^{a}$ DPNC - University of Geneva 24 quai Ernest Ansermet, CH1211 Genève 4, Switzerland

${ }^{b}$ Instituto de Astrofsica de Canarias and Universidad de La Laguna, La Laguna, Spain

${ }^{c}$ University of Trieste and INFN, Trieste, Italy

E-mail: alessandro.carosi@unige.ch

The Cherenkov Telescope Array (CTA) is a next generation ground-based very-high-energy gamma-ray observatory that will allow for observations in the $>10 \mathrm{GeV}$ range with unprecedented photon statistics and sensitivity. This will enable the investigation of the yet-marginally explored physics of short-time-scale transient events. CTA will thus become an invaluable instrument for the study of the physics of the most extreme and violent objects and their interactions with the surrounding environment. The CTA Transient program includes follow-up observations of a wide range of multi-wavelength and multi-messenger alerts, ranging from compact galactic binary systems to extragalactic events such as gamma-ray bursts (GRBs), core-collapse supernovae and bright AGN flares. In recent years, the first firm detection of GRBs by current Cherenkov telescope collaborations, the proven connection between gravitational waves and short GRBs, as well as the possible neutrino-blazar association with TXS 0506+056 have shown the importance of coordinated follow-up observations triggered by these different cosmic signals in the framework of the birth of multi-messenger astrophysics. In the next years, CTA will play a major role in these types of observations by taking advantage of its fast slewing (especially for the CTA Large Size Telescopes), large effective area and good sensitivity, opening new opportunities for time-domain astrophysics in an energy range not affected by selective absorption processes typical of other wavelengths. In this contribution we highlight the common approach adopted by the CTA Transients physics working group to perform the study of transient sources in the very-high-energy regime.

$37^{\text {th }}$ International Cosmic Ray Conference (ICRC 2021)

July 12th - 23rd, 2021

Online - Berlin, Germany

\footnotetext{
*Presenter
} 


\section{Introduction}

In recent years, the field of very-high-energy (VHE, E >100 GeV) transient astronomy has begun evolving evolving toward the observations of new sources in the context of a multi-messenger approach. The comprehension of the connection between time-domain and multi-messenger astrophysics is rapidly becoming of primary importance since VHE photons are produced in extreme, violent environments potentially associated with cosmic accelerators and therefore to the production of high-energy cosmic-rays and neutrinos. Furthermore, VHE radiation might be expected from stellar collapses and compact object mergers which power supernovae, gamma-ray bursts (GRBs) and gravitational wave (GW) emission. Transients are an integral part of the CTA Key Science Projects (KSP) [1]. A dedicated Science Working Group (Transient and multi wavelength SWG) is in place to prepare first observations (react to fast target of opportunities-ToO, define the observation program, prepare the scientific analysis, etc..) and set up the needed multi wavelength/multi-messenger connections and synergies with external facilities. The main scientific goals of the group, include the release of dedicated consortium publications focused on key topics such as GRBs, gravitational waves, neutrino ToOs, galactic transients and core-collapse supernovae. The group is also involved in other activities such as evaluating the detection prospects of serendipitous VHE transients identified via the CTA real-time analysis and the VHE transient survey, by exploring the divergent pointing capability in association with the CTA extragalactic survey KSP.

In this contribution, we will report on the general Transients KSP and each of the main subprojects (namely consortium publications) under development within the Transients SWG, their current status and planned activities.

\section{The Transients KSP}

The Transients KSP is an important part of the CTA science core program and one of its main goals is to prepare the response of CTA to a wide range of multi-wavelength and multi-messenger alerts, including GRBs, GWs and high-energy neutrinos. The sensitivity achievable to emission on short timescales and the fast response to external alerts will allow CTA to probe ultra-fast variability over early phases of transient events in unprecedented detail compared to current generation imaging atmospheric Cherenkov telescopes (IACTs) and space-based instrumentation (see Fig.1 left panel).

This would allow us to shed some light on some fundamental topics such as:

- The investigation of the physical mechanisms that drive jets and winds around neutron stars and black holes

- The study of and potential discrimination between the different emission scenarios and physical processes capable of producing the observed HE and VHE radiation from GRBs

- The origin of the ultra-high energy cosmic rays (UHECRs) and high-energy neutrinos

- The study of the physical mechanisms at the origin of GWs and their electromagnetic counterparts 


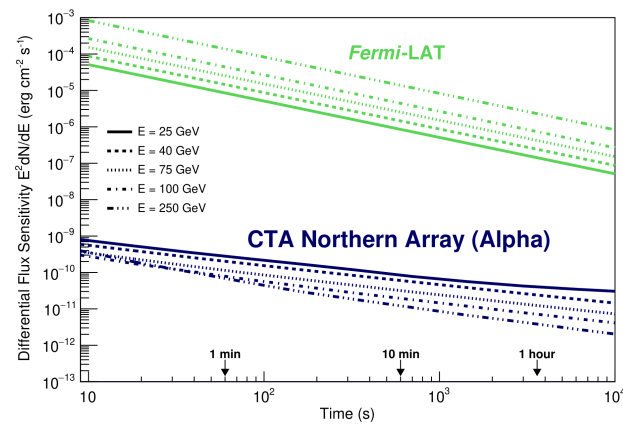

\begin{tabular}{|c|c|c|c|c|}
\hline \multicolumn{5}{|c|}{ Observation times $\left(\mathrm{h} \mathrm{yr}^{-1}\right.$ site $\left.^{-1}\right)$} \\
\hline Priority & Target class & Early phase & Years 1-2 & Years 3-10 \\
\hline 1 & GW transients & 20 & 5 & 5 \\
\hline 2 & $\mathrm{HE}$ neutrino transients & 20 & 5 & 5 \\
\hline 3 & Serendipitous detections & 100 & 25 & 25 \\
\hline 4 & GRBs & 50 & 50 & 50 \\
\hline 5 & MWL transients & 50 & 10 & 10 \\
\hline 6 & Galactic & 150 & 30 & 0 \\
\hline Prel. total observation time & & 390 & 125 & 95 \\
\hline
\end{tabular}

Figure 1: Left: Sensitivities of CTA and Fermi-LAT in their overlapping energy range as a function of observation time. Differential flux sensitivities at three energies are compared. For CTA, the proposed alpha configuration with 4 large sized telescopes (LST) and 9 medium sized telescopes (MST) in the north array is reported. From https://www.cta-observatory.org/science/cta-performance/. Right: Summary of the observation proposed in the Key Science Project proposal in [1].

Starting from these key science topics, and also using the extensive experience of current IACTs, the transient KSP is poised to optimize the CTA response to transient alerts by providing a list of detailed use cases covering a wide range of observational steps: from the receiving of the alerts to the reaction of the arrays; from data taking to the rapid feedback to the astronomical community on the VHE properties of the observed transients. A preliminary list of the proposed observations within the CTA transient KSP is reported in Fig.(1) although the outlined numbers are currently going under a major revision according to the latest, state-of-the-art knowledge derived from current IACTs follow-ups.

\section{Gamma-ray Bursts}

GRBs have always been considered a primary targets for all modern IACT telescopes. The detection of VHE gamma-ray from a number of events including GRB 180720B [2], GRB 190114C [3] and GRB 190829A [4] represents a long-awaited result and a remarkable step forward in our understanding of GRB physics. For long time, the search for VHE signals associated with GRBs posed a major challenge for IACTs from both the technical and the scientific point of view (see e.g. [5] [6]). The possibility of detecting a VHE gamma-ray signal from a GRB is indeed crucial for clarifying the poorly-known physics of these objects during the different phases of their emission, in particular during the early afterglow phase when the co-existence of forward and reverse shocks in the emitted outflow could yield a large variety of different emitting scenarios. The CTA array will routinely perform follow-up observations of GRB triggers. The estimation of the detection prospects for such observations are necessarily still preliminary and are dependent on the final array layout and performance. However, even starting with simplified assumptions about the GRB emission, the CTA Consortium already reported the possibility of detecting hundreds (or more) of photons from moderate to bright GRB allowing for a significant improvement in the photon statistics and for the possibility to have good-quality time-resolved spectra [7]. In order to achieve a step forward in the determination of CTA's prospects for GRB follow-ups, the Transient SWG is currently working on a new publication where the potential detection rate is estimated using a theoretical-based approach. 


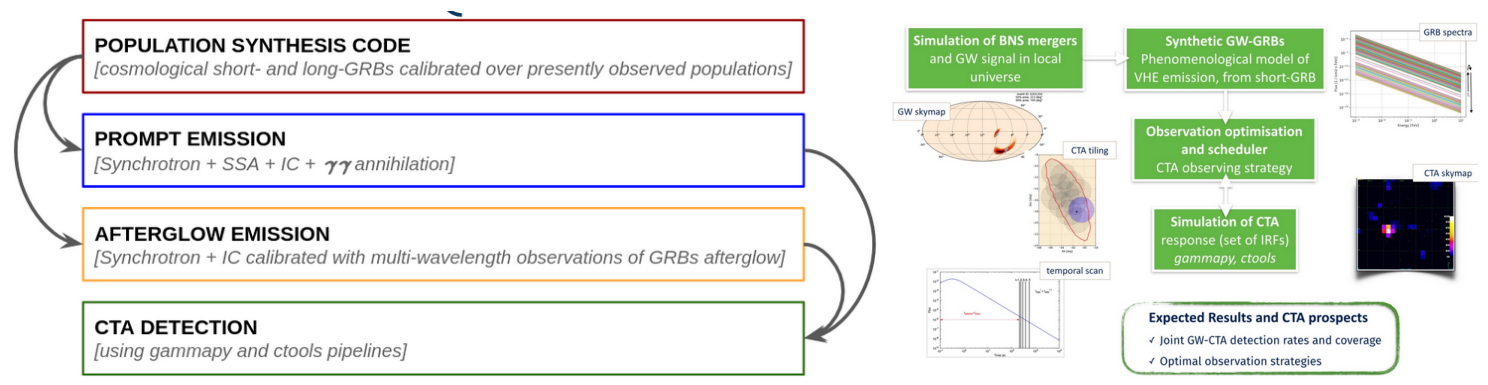

Figure 2: (Left:) Scheme of the GRB consortium publication work. Synthetic spectra and light curves are obtained by a population synthesis code and used to feed the CTA analysis pipeline. (Right:) Workflow for the GW consortium publication. After the simulation of BNS merger, a phenomenological (short) GRB is associated to it. The optimal pointing strategy is then obtained by a dedicated algorithm in order to cover efficiently the sky area of the GW source. Each pointing is then analyzed by means of the CTA analysis pipeline.

Such an approach is based on the POpulation Synthesis Theory Integrated code for Very high energy Emission (POSyTIVE) population model for GRBs [8]. The resulting population is built by considering few intrinsic properties and assumptions:

- $\mathrm{E}_{\text {peak }}$ redshift distribution

- $\mathrm{E}_{\text {peak }}-\mathrm{E}_{\text {iso }}$ correlation (Amati relation) [9]

- Bulk Lorentz factor distribution obtained by measured the time of the afterglow onset (providing the bulk Lorentz factor of the event's coasting phase)

The population obtained (for both long and short GRBs) is calibrated against a wide data set of multi-wavelength observations. In order to derive the final expected spectrum, both the prompt and the afterglow emission are simulated according to standard leptonic synchrotron and synchrotron self-Compton models [10]. The GRB spectra obtained are then used to simulate the detailed CTA response through the use of dedicated analysis pipelines based on gammapy ${ }^{1}$ and ctools ${ }^{2}$.

\section{Gravitational Waves}

The link between GWs and short GRBs (sGRBs) has been discussed widely in literature in the past and was proven definitively after the observation of the coincidence between GRB 170817A and the gravitational signal GW 170817 [11]. Although all of the GRBs detected so far by current IACTs were long GRB, sGRBs are also expected to emit VHE radiation. Nevertheless, only a hint of emission in this energy band are reported so far for sGRBs by the MAGIC telescopes [12]. However, this result, as well as the significant programs put in place by the major IACT collaborations [see e.g., 13, 14] for follow-up campaigns of GW triggers confirms the scientific potential of these observations. Thanks to its unprecedented sensitivity and the increased number of GW events expected in the near future, CTA will be able to increase the number of VHE counterpart detections

1https: //gammapy .org/

2http: //cta.irap .omp . eu/ctools/ 
providing a deeper insight into their physical processes. The detection of the potential electromagnetic counterpart is, however, challenging due to the relatively large localisation uncertainties provided by GW interferometers. Within the transient SWG, a detailed study to establish the number of possible successful CTA detections of VHE counterparts of GWs is under development [15].

In contrast to the GRB case, a purely phenomenological approach is used: a short GRB is associated to a set of simulated binary neutron star (BNS) mergers extracted from the public database GWCOSMos [16] providing the GW skymap, distance and orientation. The corresponding VHE emission is derived from the empirical correlations between the $\mathrm{X}$-ray and $\mathrm{TeV}$ luminosities as observed in the VHE GRB sample (as in GRB 190114C). The optimal pointing strategy is then obtained by a dedicated algorithm in order to cover efficiently the sky area of the GW source. Each pointing is subsequently analyzed by means of a dedicated analysis pipeline in order to provide the final outcome on a possible detection. The CTA GW follow-up program is currently being defined and implemented. The results of these studies will be the subject of a dedicated consortium publication expected by the end of 2021.

\section{Neutrino Follow-up}

The IceCube detection of the high-energy neutrino event (IC-170922A) associated with the flaring gamma-ray blazar TXS 0506+056 represents the best evidence so far seen for an astrophysical neutrino point source [17]. The extensive multi-wavelength follow-up campaign that also involved many of the currently operating IACTs, stands as an important test case for the optimization of the CTA Neutrino Target of Opportunity (NToO) program. The aim of this program is to develop a strategy for CTA follow-up of neutrino alerts to maximize the chance of detecting a VHE counterpart. Neutrino point source simulations are based on FIRESONG [18], which takes into account the cosmological evolution of different source classes and the recent results from IceCube (i.e., the measured diffuse flux of astrophysical neutrinos). These are then the input for simulating the expected VHE gamma-ray emission. Neutrinos might indeed be accompanied by VHE gamma rays produced according to typical pp and $\mathrm{p} \gamma$ models. CTA, with its fast reaction time and lower energy threshold, will enable sensitive searches for such VHE counterparts for well-localized, likely astrophysical neutrino events up to much higher redshifts than those accessible to current IACTs (Fig. 3). Preliminary results $[19,20]$ show that, for flaring blazars, CTA will be able to detect a VHE counterpart for about one third of the cases after $\sim 10$ mins of observations, with lower detection probabilities for steady neutrino sources. For more details, see the dedicated contribution at this conference [21].

\section{Galactic Transients}

Several sources in our Galaxy exhibit transient emission via different processes, such as outflows interacting with the surrounding interstellar medium, strong winds or accretion/ejection from/onto a compact object. Some of these events are energetic enough to accelerate particles via non-thermal mechanisms and produce high-energy gamma-ray emission in the $\mathrm{MeV}$ range. But the main question that arises is whether these $\mathrm{MeV}$ emitters can also be sources of VHE gamma rays. For the moment, searches for VHE emission from different types of gamma-ray emitters have not 

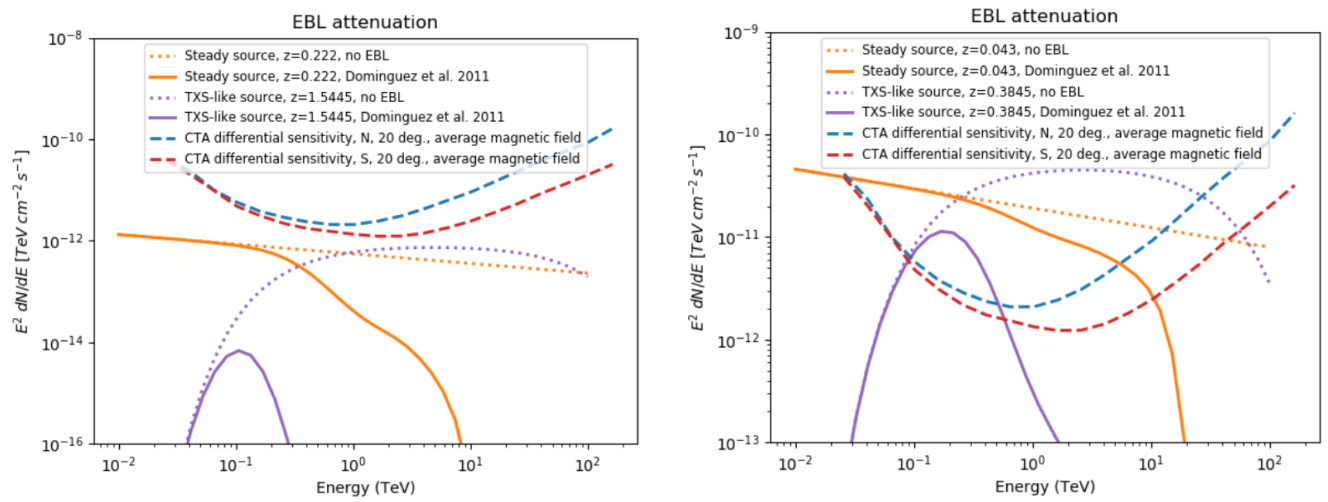

Figure 3: The energy spectra of potential neutrino sources (without and with the EBL attenuation) overlaid on the CTA differential sensitivity for the undetected (left) and detected (right) sources. CTA sensitivities correspond to the omega configuration for both the Southern (4 LSTs + 25 MSTs + 70 SSTs) and the Northern hemisphere (4 LSTs + 15 MSTs) from [20].

revealed any signal with current IACTs. CTA, with its unprecedented sensitivity to short-timescale transient events, will perform first-time detections of the VHE component of many MeV-emitters and will likely achieve serendipitous discoveries of yet unknown sources. CTA observations of these flaring objects will be triggered by external facilities such as X-ray or high-energy monitoring satellites. Serendipitous detections might also happen while performing, i.e., surveys such as that of the Galactic Plane. For the detection of Galactic transients, the real-time analysis will play a key role in the follow-up and observation strategies of these externally triggered events. Among the different types of Galactic transients, we have tested the capabilities of CTA to detect transient emission from two types of MeV-emitters: flares from pulsar wind nebulae (PWNe) and microquasars. A more detailed analysis of these and others Galactic transient sources, is reported in [22].

Flares from the Crab Nebula: PWNe are bubbles of relativistic plasma which are powered by the magnetically-driven winds of a central highly-magnetized rotating neutron star (pulsar). The Crab Nebula PWN is the brightest persistent VHE source in the TeV gamma-ray sky, and is referred to as the standard candle. AGILE, however, discovered flaring activity from this source [23], which was subsequently confirmed by Fermi-LAT [24]. We prove that CTA-N will be able to detect VHE flaring emission from the Crab Nebula down to $10 \%$ of flare intensity measured by AGILE, thanks to its wide energy range and its improved sensitivity with respect to the current generation of IACTs.

Microquasars: Microquasars are binaries composed of a compact object (which can be a black hole or a neutron star) and a companion star. Matter is accreted onto the compact object, creating an accretion disk and (eventually) generating collimated jets of plasma. We have tested whether some $\mathrm{MeV}$ emitters, such as the microquasars in the Cygnus region, can also be detected at VHE by CTA. We have tested whether the two massive microquasars Cyg X-1 and Cyg X-3, which are known MeV sources [25, 26] could also be detected with CTA-N, even if no evidence for VHE emission has been claimed by current IACTs [27, 28]. Only marginal evidence for a VHE signal was observed by the MAGIC telescopes during an 80-minute long observation in 2006 [29], coincident with a hard X-ray flare. We highlight the capability of the CTA-N array to detect transient emission from both of the microquasars, emphasizing the detection of transient emission from Cyg X-1 in 
only 30 minutes.

CTA will provide crucial insights which will help to refine emission models for microquasars and jet formation, and will reveal the mechanisms at work in flaring PWNe at VHE. Its unique sensitivity to short-time events and its large energy coverage will allow CTA to discover the VHE components of many Galactic $\mathrm{MeV}$ emitters and to reveal serendipitous detections of unknown sources.

\section{Core-Collapse Supernovae}

A core-collapse supernova (CCSNe) represents the catastrophic explosion of a massive star at the end of its life. The energy is mainly released in the form of kinetic energy of a nonrelativistic expanding outflow. In the resulting fast-moving shock wave, particles are accelerated via the first-order Fermi mechanism. The accelerated particles, interacting with the surrounding interstellar matter, might lead to the production of a gamma-ray signal up to the VHE band that can be potentially detected by CTA. A wide range of different types of CCSNe exists (IIP, IIL, IIb, IIn, etc. ) which could each have a different signature in the VHE regime. A dedicated work on the prospects for the CTA detection of such a signal has recently started within the Transients working group. A set of known CCSne will be used as a template to model the expected VHE emission in the CTA energy range. Such emission is then input to dedicated CTA analysis pipelines to study the CTA prospects for such observations during the first days/months (and possibly years) after the explosion.

\section{Acknowledgements}

This work was conducted in the context of the CTA Transients Working Group. We gratefully acknowledge financial support from the agencies and organizations listed here: http://www.ctaobservatory.org/consortium_acknowledgments.

\section{References}

[1] B. S. Archaya et al (CTA Consortium), 2019, Published by World Scientific Publishing Co., https://doi.org/10.1142/10986

[2] H.E.S.S. Collaboration: Abdalla, H. \& et al., 2019, Nature, 575, 464

[3] MAGIC Collaboration: Acciari, V. A. \& et al., 2019, Nature, 575, 455

[4] H.E.S.S. Collaboration: ATeL n.13052

[5] MAGIC Collaboration, Aleksić, J. et al., 2010, A\&A 517, 5

[6] MAGIC Collaboration, Aleksić, J. et al., 2013, MNRAS, 437, 3103

[7] Inoue, S., Granot, J., O’Brien, P. et al., 2013, APh, 43, 252

[8] Sadeh, I. et al., 2019, in 36th International Cosmic Ray Conference (ICRC 2019), PoS598 
[9] Amati, L., et al., 2002, A\&A, 390, 81

[10] R. Sari \& A. A. Esin, 2001, ApJ, 548, 787

[11] B. P. Abbott, B. P. R. Abbott, R., Abbott, T.D. et al., 2017, ApJL, 848 L12

[12] MAGIC Collaboration: Acciari, V. A. et al, 2021, Astrophys. J.908, 90, 2012.07193.

[13] H.E.S.S. Collaboration: Abdalla, H. et al., 2017, Astrophys. J. Lett.850, L22, 1710.05862

[14] H.E.S.S. Collaboration: Abdalla, H. et al., 2020, Astrophys. J. Lett.894, L16, 2004.10105.

[15] Seglar-Arroyo, M. et al., 2019, in 36th International Cosmic Ray Conference (ICRC 2019), PoS790.

[16] Patricelli, B., Stamerra, A., Razzano, M. et al. 2018, JCAP, 05, 056

[17] IceCube Collaboration, 2018, Science 361, 1378

[18] Taboada, I. et al., 2019, in 35th International Cosmic Ray Conference (ICRC 2017) PoS(ICRC2017)663

[19] Satalecka, K., Brown, A., Rosales de Le on, O. Sergijenko, et al. (CTA Consortium, FIRESONG Team), in 36th International Cosmic Ray Conference (ICRC 2019)(2019), vol. 36 of International Cosmic Ray Conference, p. 784.

[20] Bosnjak, Z., Brown, A.M., Carosi, A. at al. (CTA Consortium), 2021, Submitted to ASTRONET roadmap, arXiv:2106.03621v

[21] O. Sergijenko et al. (CTA Consortium), 2021, in 37th International Cosmic Ray Conference (ICRC 2021), contribution id.224

[22] López-Oramas, A. et al. ICRC2021, (CTA Consortium), 2021, in 37th International Cosmic Ray Conference (ICRC 2021), contribution id.224

[23] Tavani, M., Bulgarelli, A., Vittorini, V. et al., 2011, Science, Volume 331, Issue 6018

[24] Abdo .A. A. et al. (Fermi-LAT), 2011, Science 331, 739, 1011.3855

[25] Sabatini, S., Tavani, M., Striani, E., 2010, ApJL, 712, Issue 1

[26] Zanin, R. et al. 2016, A\&A, 596, id.A55

[27] MAGIC Collaboration: Aleksić, J. et al., 2010, ApJ, 721, 1

[28] MAGIC Collaboration: Ahnen, A. et al., 2017, MNRAS, 472, 3

[29] MAGIC Collaboration: Albert, A. et al., 2007, ApJL, 665, L51 


\section{Full Authors List: CTA Collaboration}

H. Abdalla ${ }^{1}$, H. Abe ${ }^{2}$, S. Abe $^{2}$, A. Abusleme ${ }^{3}$, F. Acero ${ }^{4}$, A. Acharyya ${ }^{5}$, V. Acín Portella ${ }^{6}$, K. Ackley ${ }^{7}$, R. Adam ${ }^{8}$, C. Adams ${ }^{9}$, S.S. Adhikari ${ }^{10}$, I. Aguado-Ruesga ${ }^{11}$, I. Agudo $^{12}$, R. Aguilera ${ }^{13}$, A. Aguirre-Santaella ${ }^{14}$, F. Aharonian ${ }^{15}$, A. Alberdi ${ }^{12}$, R. Alfaro ${ }^{16}$, J. Alfaro ${ }^{3}$, C. Alispach ${ }^{17}$, R. Aloisio ${ }^{18}$, R. Alves Batista ${ }^{19}$, J.-P. Amans ${ }^{20}$, L. Amati ${ }^{21}$, E. Amato ${ }^{22}$, L. Ambrogi ${ }^{18}$, G. Ambrosi $^{23}$, M. Ambrosio ${ }^{24}$, R. Ammendola ${ }^{25}$, J. Anderson ${ }^{26}$, M. Anduze $^{8}$, E.O. Angüner ${ }^{27}$, L.A. Antonelli ${ }^{28}$, V. Antonuccio ${ }^{29}$, P. Antoranz ${ }^{30}$, R. Anutarawiramkul ${ }^{31}$, J. Aragunde Gutierrez ${ }^{32}$, C. Aramo ${ }^{24}$, A. Araudo ${ }^{33,34}$, M. Araya ${ }^{35}$, A. Arbet-Engels ${ }^{36}$, C. Arcaro $^{1}$, V. Arendt ${ }^{37}$, C. Armand ${ }^{38}$, T. Armstrong ${ }^{27}$, F. Arqueros ${ }^{11}$, L. Arrabito ${ }^{39}$, B. Arsioli ${ }^{40}$, M. Artero ${ }^{41}$, K. Asano ${ }^{2}$, Y. Ascasíbar ${ }^{14}$, J. Aschersleben $^{42}$, M. Ashley ${ }^{43}$, P. Attinà ${ }^{44}$, P. Aubert ${ }^{45}$, C. B. Singh ${ }^{19}$, D. Baack ${ }^{46}$, A. Babic ${ }^{47}$, M. Backes ${ }^{48}$, V. Baena ${ }^{13}$, S. Bajtlik ${ }^{49}$, A. Baktash ${ }^{50}$, C. Balazs ${ }^{7}$, M. Balbo ${ }^{38}$, O. Ballester ${ }^{41}$, J. Ballet ${ }^{4}$, B. Balmaverde ${ }^{44}$, A. Bamba ${ }^{51}$, R. Bandiera ${ }^{22}$, A. Baquero Larriva ${ }^{11}$, P. Barai ${ }^{19}$, C. Barbier ${ }^{45}$, V. Barbosa Martins ${ }^{52}$, M. Barcelo ${ }^{53}$, M. Barkov ${ }^{54}$, M. Barnard ${ }^{1}$, L. Baroncelli ${ }^{21}$, U. Barres de Almeida ${ }^{40}$, J.A. Barrio ${ }^{11}$, D. Bastieri ${ }^{55}$, P.I. Batista ${ }^{52}$, I. Batkovic ${ }^{55}$, C. Bauer ${ }^{53}$, R. Bautista-González ${ }^{56}$, J. Baxter ${ }^{2}$, U. Becciani ${ }^{29}$, J. Becerra González ${ }^{32}$, Y. Becherini ${ }^{57}$, G. Beck ${ }^{58}$, J. Becker Tjus ${ }^{59}$, W. Bednarek ${ }^{60}$, A. Belfiore ${ }^{61}$, L. Bellizzi ${ }^{62}$, R. Belmont ${ }^{4}$, W. Benbow ${ }^{63}$, D. Berge ${ }^{52}$, E. Bernardini ${ }^{52}$, M.I. Bernardos ${ }^{55}$, K. Bernlöhr ${ }^{53}$, A. Berti ${ }^{64}$, M. Berton ${ }^{65}$, B. Bertucci ${ }^{23}$, V. Beshley ${ }^{66}$, N. Bhatt ${ }^{67}$, S. Bhattacharyya ${ }^{67}$, W. Bhattacharyya ${ }^{52}$, S. Bhattacharyya ${ }^{68}$, B. Bi ${ }^{69}$, G. Bicknell ${ }^{70}$, N. Biederbeck ${ }^{46}$, C. Bigongiari ${ }^{28}$, A. Biland ${ }^{36}$, R. Bird ${ }^{71}$, E. Bissaldi ${ }^{72}$, J. Biteau ${ }^{73}$, M. Bitossi ${ }^{74}$, O. Blanch ${ }^{41}$, M. Blank ${ }^{50}$, J. Blazek ${ }^{33}$, J. Bobin ${ }^{75}$, C. Boccato ${ }^{76}$, F. Bocchino ${ }^{77}$, C. Boehm ${ }^{78}$, M. Bohacova ${ }^{33}$, C. Boisson ${ }^{20}$, J. Boix ${ }^{41}$, J.-P. Bolle ${ }^{52}$, J. Bolmont ${ }^{79}$, G. Bonanno ${ }^{29}$, C. Bonavolontà ${ }^{24}$, L. Bonneau Arbeletche ${ }^{80}$, G. Bonnoli $^{12}$, P. Bordas ${ }^{81}$, J. Borkowski ${ }^{49}$, S. Bórquez ${ }^{35}$, R. Bose ${ }^{82}$, D. Bose ${ }^{83}$, Z. Bosnjak ${ }^{47}$, E. Bottacini5 ${ }^{55}$, M. Böttcher ${ }^{1}$, M.T. Botticella ${ }^{84}$, C. Boutonnet ${ }^{85}$, F. Bouyjou ${ }^{75}$, V. Bozhilov ${ }^{86}$, E. Bozzo ${ }^{38}$, L. Brahimi ${ }^{39}$, C. Braiding ${ }^{43}$, S. BrauNogué $^{87}$, S. Breen ${ }^{78}$, J. Bregeon ${ }^{39}$, M. Breuhaus ${ }^{53}$, A. Brill ${ }^{9}$, W. Brisken ${ }^{88}$, E. Brocato ${ }^{28}$, A.M. Brown ${ }^{5}$, K. Brügge ${ }^{46}$, P. Brun ${ }^{89}$, P. Brun ${ }^{39}$, F. Brun ${ }^{89}$, L. Brunetti ${ }^{45}$, G. Brunetti ${ }^{90}$, P. Bruno ${ }^{29}$, A. Bruno ${ }^{91}$, A. Bruzzese ${ }^{6}$, N. Bucciantini ${ }^{22}$, J. Buckley ${ }^{82}$, R. Bühler ${ }^{52}$, A. Bulgarelli ${ }^{21}$, T. Bulik ${ }^{92}$, M. Bünning $^{52}$, M. Bunse ${ }^{46}$, M. Burton ${ }^{93}$, A. Burtovoi ${ }^{76}$, M. Buscemi ${ }^{94}$, S. Buschjäger ${ }^{46}$, G. Busetto ${ }^{55}$, J. Buss ${ }^{46}$, K. Byrum $^{26}$, A. Caccianiga ${ }^{95}$, F. Cadoux ${ }^{17}$, A. Calanducci ${ }^{29}$, C. Calderón ${ }^{3}$, J. Calvo Tovar ${ }^{32}$, R. Cameron ${ }^{96}$, P. Campaña ${ }^{35}$, R. Canestrari ${ }^{91}$, F. Cangemi ${ }^{79}$, B. Cantlay ${ }^{31}$, M. Capalbi ${ }^{91}$, M. Capasso ${ }^{9}$, M. Cappi ${ }^{21}$, A. Caproni9 ${ }^{97}$, R. Capuzzo-Dolcetta ${ }^{28}$, P. Caraveo ${ }^{61}$, V. Cárdenas ${ }^{98}$, L. Cardiel ${ }^{41}$, M. Cardillo ${ }^{99}$, C. Carlile ${ }^{100}$, S. Caroff ${ }^{45}$, R. Carosi ${ }^{74}$, A. Carosi ${ }^{17}$, E. Carquín ${ }^{35}$, M. Carrère ${ }^{39}$, J.-M. Casandjian ${ }^{4}$, S. Casanova ${ }^{101,53}$, E. Cascone ${ }^{84}$, F. Cassol ${ }^{27}$, A.J. Castro-Tirado ${ }^{12}$, F. Catalani ${ }^{102}$, O. Catalano ${ }^{91}$, D. Cauz ${ }^{103}$, A. Ceccanti ${ }^{64}$, C. Celestino Silva ${ }^{80}$, S. Celli ${ }^{18}$, K. Cerny ${ }^{104}$, M. Cerruti ${ }^{85}$, E. Chabanne ${ }^{45}$, P. Chadwick ${ }^{5}$, Y. Chai ${ }^{105}$, P. Chambery ${ }^{106}$, C. Champion $^{85}$, S. Chandra ${ }^{1}$, S. Chaty ${ }^{4}$, A. Chen ${ }^{58}$, K. Cheng ${ }^{2}$, M. Chernyakova ${ }^{107}$, G. Chiaro ${ }^{61}$, A. Chiavassa ${ }^{64,108}$, M. Chikawa ${ }^{2}$, V.R. Chitnis ${ }^{109}$, J. Chudoba ${ }^{33}$, L. Chytka ${ }^{104}$, S. Cikota ${ }^{47}$, A. Circiello ${ }^{24,110}$, P. Clark ${ }^{5}$, M. Çolak ${ }^{41}$, E. Colombo ${ }^{32}$, J. Colome ${ }^{13}$, S. Colonges ${ }^{85}$, A. Comastri ${ }^{21}$, A. Compagnino ${ }^{91}$, V. Conforti ${ }^{21}$, E. Congiu ${ }^{95}$, R. Coniglione ${ }^{94}$, J. Conrad ${ }^{111}$, F. Conte ${ }^{53}$, J.L. Contreras ${ }^{11}$, P. Coppi ${ }^{112}$, R. Cornat ${ }^{8}$, J. Coronado-Blazquez ${ }^{14}$, J. Cortina ${ }^{113}$, A. Costa $^{29}$, H. Costantini ${ }^{27}$, G. Cotter ${ }^{114}$, B. Courty ${ }^{85}$, S. Covino ${ }^{95}$, S. Crestan ${ }^{61}$, P. Cristofari ${ }^{20}$, R. Crocker ${ }^{70}$, J. Croston ${ }^{115}$, K. Cubuk ${ }^{93}$, O. Cuevas ${ }^{98}$, X. Cui ${ }^{2}$, G. Cusumano ${ }^{91}$, S. Cutini ${ }^{23}$, A. D’Aì ${ }^{91}$, G. D’Amico ${ }^{116}$, F. D'Ammando ${ }^{90}$, P. D'Avanzo ${ }^{95}$, P. Da Vela ${ }^{74}$, M. Dadina ${ }^{21}$, S. Dai ${ }^{117}$, M. Dalchenko ${ }^{17}$, M. Dall' Ora ${ }^{84}$, M.K. Daniel ${ }^{63}$, J. Dauguet ${ }^{85}$, I. Davids ${ }^{48}$, J. Davies ${ }^{114}$, 
B. Dawson ${ }^{118}$, A. De Angelis ${ }^{55}$, A.E. de Araújo Carvalho ${ }^{40}$, M. de Bony de Lavergne ${ }^{45}$, V. De Caprio $^{84}$, G. De Cesare ${ }^{21}$, F. De Frondat ${ }^{20}$, E.M. de Gouveia Dal Pino ${ }^{19}$, I. de la Calle ${ }^{11}$, B. De Lotto ${ }^{103}$, A. De Luca ${ }^{61}$, D. De Martino ${ }^{84}$, R.M. de Menezes ${ }^{19}$, M. de Naurois ${ }^{8}$, E. de Oña Wilhelmi ${ }^{13}$, F. De Palma ${ }^{64}$, F. De Persio ${ }^{119}$, N. de Simone ${ }^{52}$, V. de Souza ${ }^{80}$, M. Del Santo $^{91}$, M.V. del Valle ${ }^{19}$, E. Delagnes ${ }^{75}$, G. Deleglise ${ }^{45}$, M. Delfino Reznicek ${ }^{6}$, C. Delgado ${ }^{113}$, A.G. Delgado Giler ${ }^{80}$, J. Delgado Mengual ${ }^{6}$, R. Della $\mathrm{Ceca}^{95}$, M. Della Valle ${ }^{84}$, D. della Volpe ${ }^{17}$, D. Depaoli ${ }^{64,108}$, D. Depouez ${ }^{27}$, J. Devin ${ }^{85}$, T. Di Girolamo ${ }^{24,110}$, C. Di Giulio ${ }^{25}$, A. Di Piano ${ }^{21}$, F. Di Pierro $^{64}$, L. Di Venere ${ }^{120}$, C. Díaz ${ }^{113}$, C. Díaz-Bahamondes ${ }^{3}$, C. Dib ${ }^{35}$, S. Diebold ${ }^{69}$, S. Digel ${ }^{96}$, R. Dima ${ }^{55}$, A. Djannati-Atai ${ }^{85}$, J. Djuvsland ${ }^{116}$, A. Dmytriiev ${ }^{20}$, K. Docher ${ }^{9}$, A. Domínguez ${ }^{11}$, D. Dominis Prester ${ }^{121}$, A. Donath ${ }^{53}$, A. Donini ${ }^{41}$, D. Dorner ${ }^{122}$, M. Doro ${ }^{55}$, R.d.C. dos Anjos ${ }^{123}$, J.-L. Dournaux ${ }^{20}$, T. Downes ${ }^{107}$, G. Drake ${ }^{26}$, H. Drass ${ }^{3}$, D. Dravins ${ }^{100}$, C. Duangchan ${ }^{31}$, A. Duara ${ }^{124}$, G. Dubus ${ }^{125}$, L. Ducci ${ }^{69}$, C. Duffy ${ }^{124}$, D. Dumora ${ }^{106}$, K. Dundas Morå ${ }^{111}$, A. Durkalec ${ }^{126}$, V.V. Dwarkadas ${ }^{127}$, J. Ebr ${ }^{33}$, C. Eckner ${ }^{45}$, J. Eder ${ }^{105}$, A. Ederoclite ${ }^{19}$, E. Edy ${ }^{8}$, K. Egberts ${ }^{128}$, S. Einecke ${ }^{118}$, J. Eisch ${ }^{129}$, C. Eleftheriadis ${ }^{130}$, D. Elsässer ${ }^{46}$, G. Emery ${ }^{17}$, D. Emmanoulopoulos ${ }^{115}$, J.-P. Ernenwein ${ }^{27}$, M.Errando ${ }^{82}$, P. Escarate ${ }^{35}$, J. Escudero ${ }^{12}$, C. Espinoza ${ }^{3}$, S. Ettori ${ }^{21}$, A. Eungwanichayapant ${ }^{31}$ P. Evans ${ }^{124}$, C. Evoli ${ }^{18}$, M. Fairbairn ${ }^{131}$, D. Falceta-Goncalves ${ }^{132}$, A. Falcone ${ }^{133}$, V. Fallah Ramazani ${ }^{65}$, R. Falomo ${ }^{76}$, K. Farakos ${ }^{134}$, G. Fasola ${ }^{20}$, A. Fattorini ${ }^{46}$, Y. Favre ${ }^{17}$, R. Fedora ${ }^{135}$, E. Fedorova ${ }^{136}$, S. Fegan ${ }^{8}$, K. Feijen ${ }^{118}$, Q. Feng ${ }^{9}$, G. Ferrand ${ }^{54}$, G. Ferrara ${ }^{94}$, O. Ferreira ${ }^{8}$, M. Fesquet ${ }^{75}$, E. Fiandrini ${ }^{23}$, A. Fiasson ${ }^{45}$, M. Filipovic ${ }^{117}$, D. Fink ${ }^{105}$, J.P. Finley ${ }^{137}$, V. Fioretti ${ }^{21}$, D.F.G. Fiorillo ${ }^{24,110}$, M. Fiorini ${ }^{61}$, S. Flis ${ }^{52}$, H. Flores ${ }^{20}$, L. Foffano ${ }^{17}$, C. Föhr ${ }^{53}$, M.V. Fonseca ${ }^{11}$, L. Font ${ }^{138}$, G. Fontaine ${ }^{8}$, O. Fornieri ${ }^{52}$, P. Fortin ${ }^{63}$, L. Fortson ${ }^{88}$, N. Fouque ${ }^{45}$, A. Fournier ${ }^{106}$, B. Fraga ${ }^{40}$, A. Franceschini ${ }^{76}$, F.J. Franco ${ }^{30}$, A. Franco Ordovas ${ }^{32}$, L. Freixas Coromina ${ }^{113}$, L. Fresnillo ${ }^{30}$, C. Fruck ${ }^{105}$, D. Fugazza ${ }^{95}$, Y. Fujikawa ${ }^{139}$, Y. Fujita ${ }^{2}$, S. Fukami ${ }^{2}$, Y. Fukazawa ${ }^{140}$, Y. Fukui ${ }^{141}$, D. Fulla ${ }^{52}$, S. Funk ${ }^{142}$, A. Furniss ${ }^{143}$, O. Gabella ${ }^{39}$, S. Gabici ${ }^{85}$, D. Gaggero ${ }^{14}$, G. Galanti $^{61}$, G. Galaz $^{3}$, P. Galdemard ${ }^{144}$, Y. Gallant ${ }^{39}$, D. Galloway ${ }^{7}$, S. Gallozzi ${ }^{28}$, V. Gammaldi ${ }^{14}$, R. Garcia ${ }^{41}$, E. Garcia ${ }^{45}$, E. García ${ }^{13}$, R. Garcia López ${ }^{32}$, M. Garczarczyk ${ }^{52}$, F. Gargano ${ }^{120}$, C. Gargano ${ }^{91}$, S. Garozzo ${ }^{29}$, D. Gascon ${ }^{81}$, T. Gasparetto ${ }^{145}$, D. Gasparrini ${ }^{25}$, H. Gasparyan ${ }^{52}$, M. Gaug ${ }^{138}$, N. Geffroy ${ }^{45}$, A. Gent ${ }^{146}$, S. Germani ${ }^{76}$, L. Gesa ${ }^{13}$, A. Ghalumyan ${ }^{147}$, A. Ghedina ${ }^{148}$, G. Ghirlanda ${ }^{95}$, F. Gianotti ${ }^{21}$, S. Giarrusso ${ }^{91}$, M. Giarrusso ${ }^{94}$, G. Giavitto $^{52}$, B. Giebels ${ }^{8}$, N. Giglietto ${ }^{72}$, V. Gika ${ }^{134}$, F. Gillardo ${ }^{45}$, R. Gimenes ${ }^{19}$, F. Giordano ${ }^{149}$, G. Giovannini $^{90}$, E. Giro ${ }^{76}$, M. Giroletti ${ }^{90}$, A. Giuliani ${ }^{61}$, L. Giunti ${ }^{85}$, M. Gjaja ${ }^{9}$, J.-F. Glicenstein ${ }^{89}$, P. Gliwny ${ }^{60}$, N. Godinovic ${ }^{150}$, H. Göksu ${ }^{53}$, P. Goldoni ${ }^{85}$, J.L. Gómez ${ }^{12}$, G. Gómez-Vargas ${ }^{3}$, M.M. González ${ }^{16}$, J.M. González ${ }^{151}$, K.S. Gothe ${ }^{109}$, D. Götz ${ }^{4}$, J. Goulart Coelho ${ }^{123}$, K. Gourgouliatos ${ }^{5}$, T. Grabarczyk ${ }^{152}$, R. Graciani ${ }^{81}$, P. Grandi ${ }^{21}$, G. Grasseau ${ }^{8}$, D. Grasso ${ }^{74}$, A.J. Green ${ }^{78}$, D. Green ${ }^{105}$, J. Green ${ }^{28}$, T. Greenshaw ${ }^{153}$, I. Grenier ${ }^{4}$, P. Grespan ${ }^{55}$, A. Grillo ${ }^{29}$, M.-H. Grondin ${ }^{106}$, J. Grube ${ }^{131}$, V. Guarino ${ }^{26}$, B. Guest ${ }^{37}$, O. Gueta ${ }^{52}$, M. Gündüz ${ }^{59}$, S. Gunji ${ }^{154}$, A. Gusdorf ${ }^{20}$, G. Gyuk ${ }^{155}$, J. Hackfeld ${ }^{59}$, D. Hadasch ${ }^{2}$, J. Haga ${ }^{139}$, L. Hagge ${ }^{52}$, A. Hahn ${ }^{105}$, J.E. Hajlaoui ${ }^{85}$, H. Hakobyan ${ }^{35}$,

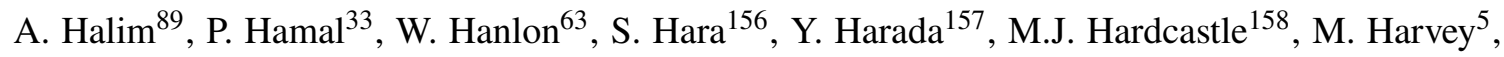
K. Hashiyama ${ }^{2}$, T. Hassan Collado ${ }^{113}$, T. Haubold ${ }^{105}$, A. Haupt ${ }^{52}$, U.A.Hautmann ${ }^{159}$, M. Havelka ${ }^{33}$, K. Hayashi ${ }^{141}$, K. Hayashi ${ }^{160}$, M. Hayashida ${ }^{161}$, H. He ${ }^{54}$, L. Heckmann ${ }^{105}$, M. Heller ${ }^{17}$, J.C. Helo ${ }^{35}$, F. Henault ${ }^{125}$, G. Henri ${ }^{125}$, G. Hermann ${ }^{53}$, R. Hermel ${ }^{45}$, S. Hernández Cadena ${ }^{16}$, J. Herrera Llorente $^{32}$, A. Herrero ${ }^{32}$, O. Hervet ${ }^{143}$, J. Hinton ${ }^{53}$, A. Hiramatsu ${ }^{157}$, N. Hiroshima ${ }^{54}$, K. Hirotani ${ }^{2}$, B. Hnatyk ${ }^{136}$, R. Hnatyk ${ }^{136}$, J.K. Hoang ${ }^{11}$, D. Hoffmann ${ }^{27}$, W. Hofmann ${ }^{53}$, C. Hoischen ${ }^{128}$, J. Holder ${ }^{162}$, M. Holler ${ }^{163}$, B. Hona ${ }^{164}$, D. Horan ${ }^{8}$, J. Hörandel ${ }^{165}$, D. Horns ${ }^{50}$, P. Horvath ${ }^{104}$, 
J. Houles ${ }^{27}$, T. Hovatta ${ }^{65}$, M. Hrabovsky ${ }^{104}$, D. Hrupec ${ }^{166}$, Y. Huang ${ }^{135}$, J.-M. Huet ${ }^{20}$, G. Hughes ${ }^{159}$,

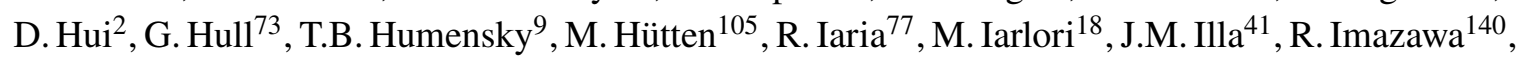
D. Impiombato ${ }^{91}$, T. Inada ${ }^{2}$, F. Incardona ${ }^{29}$, A. Ingallinera ${ }^{29}$, Y. Inome ${ }^{2}$, S. Inoue ${ }^{54}$, T. Inoue ${ }^{141}$, Y. Inoue ${ }^{167}$, A. Insolia ${ }^{120,94}$, F. Iocco ${ }^{24,110}$, K. Ioka ${ }^{168}$, M. Ionica ${ }^{23}$, M. Iori ${ }^{119}$, S. Iovenitti ${ }^{95}$, A. Iriarte ${ }^{16}$, K. Ishio ${ }^{105}$, W. Ishizaki ${ }^{168}$, Y. Iwamura ${ }^{2}$, C. Jablonski ${ }^{105}$, J. Jacquemier ${ }^{45}$, M. Jacquemont ${ }^{45}$, M. Jamrozy ${ }^{169}$, P. Janecek ${ }^{33}$, F. Jankowsky ${ }^{170}$, A. Jardin-Blicq ${ }^{31}$, C. Jarnot ${ }^{87}$, P. Jean ${ }^{87}$, I. Jiménez Martínez $^{113}$, W. Jin ${ }^{171}$, L. Jocou ${ }^{125}$, N. Jordana ${ }^{172}$, M. Josselin ${ }^{73}$, L. Jouvin ${ }^{41}$, I. Jung-Richardt ${ }^{142}$, F.J.P.A. Junqueira ${ }^{19}$, C. Juramy-Gilles ${ }^{79}$, J. Jurysek ${ }^{38}$, P. Kaaret ${ }^{173}$, L.H.S. Kadowaki ${ }^{19}$, M. Kagaya ${ }^{2}$, O. Kalekin ${ }^{142}$, R. Kankanyan ${ }^{53}$, D. Kantzas ${ }^{174}$, V. Karas ${ }^{34}$, A. Karastergiou ${ }^{114}$, S. Karkar ${ }^{79}$, E. Kasai ${ }^{48}$, J. Kasperek ${ }^{175}$, H. Katagiri ${ }^{176}$, J. Kataoka ${ }^{177}$, K. Katarzyński ${ }^{178}$, S. Katsuda ${ }^{179}$, U. Katz ${ }^{142}$, N. Kawanaka ${ }^{180}$, D. Kazanas ${ }^{130}$, D. Kerszberg ${ }^{41}$, B. Khélifi ${ }^{85}$, M.C. Kherlakian ${ }^{52}$, T.P. Kian ${ }^{181}$, D.B. Kieda ${ }^{164}$, T. Kihm ${ }^{53}$, S. Kim ${ }^{3}$, S. Kimeswenger ${ }^{163}$, S. Kisaka ${ }^{140}$, R. Kissmann ${ }^{163}$, R. Kleijwegt ${ }^{135}$, T. Kleiner ${ }^{52}$, G. Kluge ${ }^{10}$, W. Kluźniak ${ }^{49}$, J. Knapp ${ }^{52}$, J. Knödlseder ${ }^{87}$, A. Kobakhidze ${ }^{78}$, Y. Kobayashi ${ }^{2}$, B. Koch ${ }^{3}$, J. Kocot ${ }^{152}$, K. Kohri ${ }^{182}$, K. Kokkotas ${ }^{69}$, N. Kominn', A. Kong ${ }^{2}$, K. Kosack ${ }^{4}$, G. Kowal ${ }^{132}$, F. Krack ${ }^{52}$, M. Krause ${ }^{52}$, F. Krennrich ${ }^{129}$, M. Krumholz ${ }^{70}$, H. Kubo ${ }^{180}$, V. Kudryavtsev ${ }^{183}$, S. Kunwar ${ }^{53}$, Y. Kuroda ${ }^{139}$, J. Kushida ${ }^{157}$, P. Kushwaha ${ }^{19}$, A. La Barbera ${ }^{91}$, N. La Palombara ${ }^{61}$, V. La Parola ${ }^{91}$, G. La Rosa ${ }^{91}$, R. Lahmann ${ }^{142}$, G. Lamanna ${ }^{45}$, A. Lamastra ${ }^{28}$, M. Landoni ${ }^{95}$, D. Landriu ${ }^{4}$, R.G. Lang ${ }^{80}$, J. Lapington ${ }^{124}$, P. Laporte ${ }^{20}$, P. Lason ${ }^{152}$, J. Lasuik ${ }^{37}$, J. Lazendic-Galloway ${ }^{7}$, T. Le Flour ${ }^{45}$, P. Le Sidaner ${ }^{20}$, S. Leach ${ }^{124}$, A. Leckngam ${ }^{31}$, S.-H. Lee ${ }^{180}$, W.H. Lee ${ }^{16}$, S. Lee ${ }^{118}$, M.A. Leigui de Oliveira ${ }^{184}$, A. Lemière ${ }^{85}$, M. Lemoine-Goumard ${ }^{106}$, J.-P. Lenain ${ }^{79}$, F. Leone ${ }^{94,185}$, V. Leray ${ }^{8}$, G. Leto $^{29}$, F. Leuschner ${ }^{69}$, C. Levy ${ }^{79,20}$, R. Lindemann ${ }^{52}$, E. Lindfors ${ }^{65}$, L. Linhoff ${ }^{46}$, I. Liodakis ${ }^{65}$, A. Lipniacka ${ }^{116}$, S. Lloyd ${ }^{5}$, M. Lobo ${ }^{113}$, T. Lohse $^{186}$, S. Lombardi ${ }^{28}$, F. Longo ${ }^{145}$, A. Lopez ${ }^{32}$, M. López ${ }^{11}$, R. López-Coto ${ }^{55}$, S. Loporchio ${ }^{149}$, F. Louis ${ }^{75}$, M. Louys ${ }^{20}$, F. Lucarelli ${ }^{28}$, D. Lucchesi ${ }^{55}$, H. Ludwig Boudi ${ }^{39}$, P.L. Luque-Escamilla ${ }^{56}$, E. Lyard $^{38}$, M.C. Maccarone ${ }^{91}$, T. Maccarone ${ }^{187}$, E. Mach ${ }^{101}$, A.J. Maciejewski ${ }^{188}$, J. Mackey ${ }^{15}$, G.M. Madejski ${ }^{96}$, P. Maeght ${ }^{39}$, C. Maggio ${ }^{138}$, G. Maier ${ }^{52}$, A. Majczyna ${ }^{126}$, P. Majumdar ${ }^{83,2}$, M. Makariev ${ }^{189}$, M. Mallamaci ${ }^{55}$, R. Malta Nunes de Almeida ${ }^{184}$, S. Maltezos ${ }^{134}$, D. Malyshev ${ }^{142}$, D. Malyshev ${ }^{69}$, D. Mandat ${ }^{33}$, G. Maneva ${ }^{189}$, M. Manganaro ${ }^{121}$, G. Manicò ${ }^{94}$, P. Manigot ${ }^{8}$, K. Mannheim ${ }^{122}$, N. Maragos ${ }^{134}$, D. Marano ${ }^{29}$, M. Marconi ${ }^{84}$, A. Marcowith ${ }^{39}$, M. Marculewicz ${ }^{190}$, B. Marčun ${ }^{68}$,

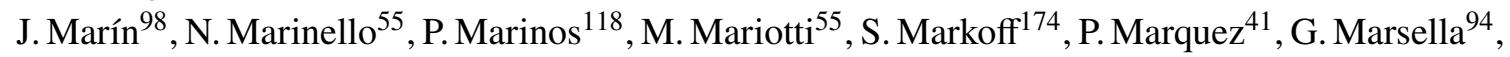
J. Martí ${ }^{56}$, J.-M. Martin ${ }^{20}$, P. Martin ${ }^{87}$, O. Martinez ${ }^{30}$, M. Martínez $^{41}$, G. Martínez $^{113}$, O. Martínez $^{41}$, H. Martínez-Huerta ${ }^{80}$, C. Marty ${ }^{87}$, R. Marx ${ }^{53}$, N. Masetti ${ }^{21,151}$, P. Massimino ${ }^{29}$, A. Mastichiadis ${ }^{191}$, H. Matsumoto ${ }^{167}$, N. Matthews ${ }^{164}$, G. Maurin ${ }^{45}$, W. Max-Moerbeck ${ }^{192}$, N. Maxted ${ }^{43}$, D. Mazin ${ }^{2,105}$, M.N. Mazziotta ${ }^{120}$, S.M. Mazzola ${ }^{77}$, J.D. Mbarubucyeye ${ }^{52}$, L. Mc Comb ${ }^{5}$, I. McHardy ${ }^{115}$, S. McKeague ${ }^{107}$, S. McMuldroch ${ }^{63}$, E. Medina ${ }^{64}$, D. Medina Miranda ${ }^{17}$, A. Melandri ${ }^{95}$, C. Melioli ${ }^{19}$, D. Melkumyan ${ }^{52}$, S. Menchiari ${ }^{62}$, S. Mender ${ }^{46}$, S. Mereghetti ${ }^{61}$, G. Merino Arévalo ${ }^{6}$, E. Mestre ${ }^{13}$, J.-L. Meunier ${ }^{79}$, T. Meures ${ }^{135}$, M. Meyer ${ }^{142}$, S. Micanovic ${ }^{121}$, M. Miceli ${ }^{77}$, M. Michailidis ${ }^{69}$, J. Michałowski ${ }^{101}$, T. Miener ${ }^{11}$, I. Mievre ${ }^{45}$, J. Miller ${ }^{35}$, I.A. Minaya ${ }^{153}$, T. Mineo ${ }^{91}$, M. Minev ${ }^{189}$, J.M. Miranda ${ }^{30}$, R. Mirzoyan ${ }^{105}$, A. Mitchell ${ }^{36}$, T. Mizuno ${ }^{193}$, B. Mode ${ }^{135}$, R. Moderski ${ }^{49}$, L. Mohrmann ${ }^{142}$, E. Molina ${ }^{81}$, E. Molinari ${ }^{148}$, T. Montaruli ${ }^{17}$, I. Monteiro ${ }^{45}$, C. Moore ${ }^{124}$, A. Moralejo ${ }^{41}$, D. MorcuendeParrilla $^{11}$, E. Moretti ${ }^{41}$, L. Morganti ${ }^{64}$, K. Mori ${ }^{194}$, P. Moriarty ${ }^{15}$, K. Morik ${ }^{46}$, G. Morlino ${ }^{22}$, P. Morris ${ }^{114}$, A. Morselli ${ }^{25}$, K. Mosshammer ${ }^{52}$, P. Moya ${ }^{192}$, R. Mukherjee ${ }^{9}$, J. Muller ${ }^{8}$, C. Mundell ${ }^{172}$,

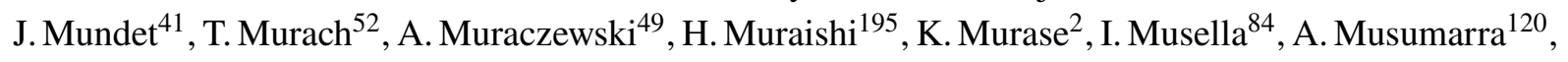


A. Nagai ${ }^{17}$, N. Nagar ${ }^{196}$, S. Nagataki ${ }^{54}$, T. Naito ${ }^{156}$, T. Nakamori ${ }^{154}$, K. Nakashima ${ }^{142}$, K. Nakayama ${ }^{51}$, N. Nakhjiri ${ }^{13}$, G. Naletto ${ }^{55}$, D. Naumann ${ }^{52}$, L. Nava ${ }^{95}$, R. Navarro ${ }^{174}$, M.A. Nawaz ${ }^{132}$, H. Ndiyavala $^{1}$, D. Neise ${ }^{36}$, L. Nellen ${ }^{16}$, R. Nemmen ${ }^{19}$, M. Newbold ${ }^{164}$, N. Neyroud ${ }^{45}$, K. Ngernphat ${ }^{31}$, T. Nguyen Trung $^{73}$, L. Nicastro ${ }^{21}$, L. Nickel ${ }^{46}$, J. Niemiec ${ }^{101}$, D. Nieto ${ }^{11}$, M. Nievas ${ }^{32}$, C. Nigro ${ }^{41}$, M. Nikołajuk ${ }^{190}$, D. Ninci ${ }^{41}$, K. Nishijima ${ }^{157}$, K. Noda ${ }^{2}$, Y. Nogami ${ }^{176}$, S. Nolan ${ }^{5}$, R. Nomura ${ }^{2}$, R. Norris ${ }^{117}$, D. Nosek ${ }^{197}$, M. Nöthe ${ }^{46}$, B. Novosyadlyj ${ }^{198}$, V. Novotny ${ }^{197}$, S. Nozaki ${ }^{180}$, F. Nunio $^{144}$, P. O’ Brien $^{124}$, K. Obara ${ }^{176}$, R. Oger ${ }^{85}$, Y. Ohira ${ }^{51}$, M. Ohishi ${ }^{2}$, S. Ohm ${ }^{52}$, Y. Ohtani ${ }^{2}$, T. Oka ${ }^{180}$, N. Okazaki ${ }^{2}$, A. Okumura ${ }^{139,199}$, J.-F. Olive ${ }^{87}$, C. Oliver $^{30}$, G. Olivera ${ }^{52}$, B. Olmi ${ }^{22}$, R.A. Ong ${ }^{71}$, M. Orienti ${ }^{90}$, R. Orito ${ }^{200}$, M. Orlandini ${ }^{21}$, S. Orlando ${ }^{77}$, E. Orlando ${ }^{145}$, J.P. Osborne ${ }^{124}$, M. Ostrowski ${ }^{169}$, N. Otte ${ }^{146}$, E. Ovcharov ${ }^{86}$, E. Owen ${ }^{2}$, I. Oya ${ }^{159}$, A. Ozieblo ${ }^{152}$, M. Padovani ${ }^{22}$, I. Pagano ${ }^{29}$, A. Pagliaro ${ }^{91}$, A. Paizis ${ }^{61}$, M. Palatiello ${ }^{145}$, M. Palatka ${ }^{33}$, E. Palazzi ${ }^{21}$, J.-L. Panazol ${ }^{45}$, D. Paneque ${ }^{105}$, B. Panes $^{3}$, S. Panny ${ }^{163}$, F.R. Pantaleo ${ }^{72}$, M. Panter ${ }^{53}$, R. Paoletti ${ }^{62}$, M. Paolillo ${ }^{24110}$, A. Papitto ${ }^{28}$, A. Paravac ${ }^{122}$, J.M. Paredes ${ }^{81}$, G. Pareschi ${ }^{95}$, N. Park ${ }^{127}$, N. Parmiggiani ${ }^{21}$, R.D. Parsons ${ }^{186}$, P. Paśko ${ }^{201}$, S. Patel ${ }^{52}$, B. Patricellii ${ }^{28}$, G. Pauletta ${ }^{103}$, L. Pavletić ${ }^{121}$, S. Pavy ${ }^{8}$, A. Pe'er ${ }^{105}$, M. Pech $^{33}$, M. Pecimotika ${ }^{121}$, M.G. Pellegriti ${ }^{120}$, P. Peñil Del Campo ${ }^{11}$, M. Penno ${ }^{52}$, A. Pepato ${ }^{55}$, S. Perard ${ }^{106}$, C. Perennes ${ }^{55}$, G. Peres ${ }^{77}$, M. Peresano ${ }^{4}$, A. Pérez-Aguilera ${ }^{11}$, J. Pérez-Romero ${ }^{14}$, M.A. PérezTorres $^{12}$, M. Perri ${ }^{28}$, M. Persic ${ }^{103}$, S. Petrera ${ }^{18}$, P.-O. Petrucci ${ }^{125}$, O. Petruk ${ }^{66}$, B. Peyaud ${ }^{89}$, K. Pfrang ${ }^{52}$, E. Pian $^{21}$, G. Piano ${ }^{99}$, P. Piatteli ${ }^{94}$, E. Pietropaolo ${ }^{18}$, R. Pillera ${ }^{149}$, B. Pilszyk ${ }^{101}$, D. Pimentel ${ }^{202}$, F. Pintore ${ }^{91}$, C. Pio García ${ }^{41}$, G. Pirola ${ }^{64}$, F. Piron ${ }^{39}$, A. Pisarski ${ }^{190}$, S. Pita ${ }^{85}$, M. Pohl ${ }^{128}$, V. Poireau ${ }^{45}$, P. Poledrelli ${ }^{159}$, A. Pollo ${ }^{126}$, M. Polo ${ }^{113}$, C. Pongkitivanichkul ${ }^{31}$, J. Porthault ${ }^{144}$,

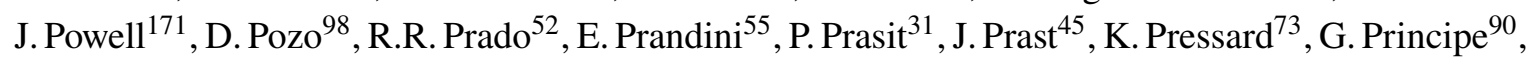
C. Priyadarshi ${ }^{41}$, N. Produit ${ }^{38}$, D. Prokhorov ${ }^{174}$, H. Prokoph ${ }^{52}$, M. Prouza ${ }^{33}$, H. Przybilski $^{101}$, E. Pueschel ${ }^{52}$, G. Pühlhofer ${ }^{69}$, I. Puljak ${ }^{150}$, M.L. Pumo ${ }^{94}$, M. Punch ${ }^{85,57}$, F. Queiroz ${ }^{203}$, J. Quinn ${ }^{204}$, A. Quirrenbach ${ }^{170}$, S. Rainò ${ }^{149}$, P.J. Rajda ${ }^{175}$, R. Rando ${ }^{55}$, S. Razzaque ${ }^{205}$, E. Rebert ${ }^{20}$, S. Recchia ${ }^{85}$, P. Reichherzer ${ }^{59}$, O. Reimer ${ }^{163}$, A. Reimer ${ }^{163}$, A. Reisenegger ${ }^{3,206}$, Q. Remy ${ }^{53}$, M. Renaud ${ }^{39}$, T. Reposeur ${ }^{106}$, B. Reville ${ }^{53}$, J.-M. Reymond ${ }^{75}$, J. Reynolds ${ }^{15}$, W. Rhode ${ }^{46}$, D. Ribeiro ${ }^{9}$, M. Ribó ${ }^{81}$, G. Richards ${ }^{162}$, T. Richtler ${ }^{196}$, J. Rico ${ }^{41}$, F. Rieger ${ }^{53}$, L. Riitano ${ }^{135}$, V. Ripepi ${ }^{84}$, M. Riquelme ${ }^{192}$, D. Riquelme ${ }^{35}$, S. Rivoire ${ }^{39}$, V. Rizi ${ }^{18}$, E. Roache ${ }^{63}$, B. Röben ${ }^{159}$, M. Roche ${ }^{106}$, J. Rodriguez ${ }^{4}$, G. Rodriguez Fernandez ${ }^{25}$, J.C. Rodriguez Ramirez ${ }^{19}$, J.J. Rodríguez Vázquez ${ }^{113}$, F. Roepke ${ }^{170}$, G. Rojas $^{207}$, L. Romanato ${ }^{55}$, P. Romano ${ }^{95}$, G. Romeo ${ }^{29}$, F. Romero Lobato ${ }^{11}$, C. Romoli ${ }^{53}$, M. Roncadelli ${ }^{103}$, S. Ronda ${ }^{30}$, J. Rosado ${ }^{11}$, A. Rosales de Leon ${ }^{5}$, G. Rowell ${ }^{118}$, B. Rudak ${ }^{49}$, A. Rugliancich ${ }^{74}$, J.E. Ruíz del Mazo ${ }^{12}$, W. Rujopakarn ${ }^{31}$, C. Rulten ${ }^{5}$, C. Russell ${ }^{3}$, F. Russo ${ }^{21}$, I. Sadeh ${ }^{52}$, E. Sæther Hatlen ${ }^{10}$, S. Safi-Harb ${ }^{37}$, L. Saha ${ }^{11}$, P. Saha ${ }^{208}$, V. Sahakian ${ }^{147}$, S. Sailer ${ }^{53}$, T. Saito ${ }^{2}$, N. Sakaki ${ }^{54}$, S. Sakurai ${ }^{2}$, F. Salesa Greus ${ }^{101}$, G. Salina ${ }^{25}$, H. Salzmann ${ }^{69}$, D. Sanchez ${ }^{45}$, M. Sánchez-Conde ${ }^{14}$, H. Sandaker ${ }^{10}$, A. Sandoval ${ }^{16}$, P. Sangiorgi ${ }^{91}$, M. Sanguillon ${ }^{39}$, H. Sano ${ }^{2}$, M. Santander ${ }^{171}$, A. Santangelo ${ }^{69}$, E.M. Santos ${ }^{202}$, R. Santos-Lima ${ }^{19}$, A. Sanuy ${ }^{81}$, L. Sapozhnikov ${ }^{96}$, T. Saric ${ }^{150}$, S. Sarkar ${ }^{114}$, H. Sasaki ${ }^{157}$, N. Sasaki ${ }^{179}$, K. Satalecka ${ }^{52}$, Y. Sato ${ }^{209}$, F.G. Saturni ${ }^{28}$, M. Sawada ${ }^{54}$, U. Sawangwit ${ }^{31}$, J. Schaefer ${ }^{142}$, A. Scherer ${ }^{3}$, J. Scherpenberg ${ }^{105}$, P. Schipani ${ }^{84}$, B. Schleicher ${ }^{122}$, J. Schmoll ${ }^{5}$, M. Schneider ${ }^{143}$, H. Schoorlemmer ${ }^{53}$, P. Schovanek ${ }^{33}$, F. Schussler ${ }^{89}$, B. Schwab ${ }^{142}$, U. Schwanke ${ }^{186}$, J. Schwarz ${ }^{95}$, T. Schweizer ${ }^{105}$, E. Sciacca ${ }^{29}$, S. Scuderi61 ${ }^{6}$, M. Seglar Arroyo $^{45}$, A. Segreto ${ }^{91}$, I. Seitenzahl ${ }^{43}$, D. Semikoz ${ }^{85}$, O. Sergijenko ${ }^{136}$, J.E. Serna Franco ${ }^{16}$, M. Servillat ${ }^{20}$, K. Seweryn ${ }^{201}$, V. Sguera ${ }^{21}$, A. Shalchi ${ }^{37}$, R.Y. Shang ${ }^{71}$, P. Sharma ${ }^{73}$, R.C. Shellard ${ }^{40}$, L. Sidolii ${ }^{61}$, J. Sieiro ${ }^{81}$, H. Siejkowski ${ }^{152}$, J. Silk ${ }^{114}$, A. Sillanpää ${ }^{65}$, B.B. Singh ${ }^{109}$, K.K. Singh ${ }^{210}$, 


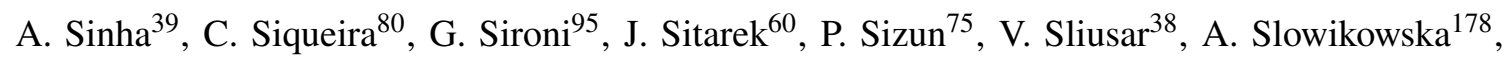
D. Sobczyńska ${ }^{60}$, R.W. Sobrinho ${ }^{184}$, H. Sol ${ }^{20}$, G. Sottile ${ }^{91}$, H. Spackman ${ }^{114}$, A. Specovius ${ }^{142}$, S. Spencer ${ }^{114}$, G. Spengler ${ }^{186}$, D. Spiga ${ }^{95}$, A. Spolon ${ }^{55}$, W. Springer ${ }^{164}$, A. Stamerra ${ }^{28}$, S. Stanič ${ }^{68}$, R. Starling ${ }^{124}$, L. Stawarz ${ }^{169}$, R. Steenkamp ${ }^{48}$, S. Stefanik ${ }^{197}$, C. Stegmann ${ }^{128}$, A. Steiner ${ }^{52}$, S. Steinmassl ${ }^{53}$, C. Stella ${ }^{103}$, C. Steppa ${ }^{128}$, R. Sternberger ${ }^{52}$, M. Sterzel ${ }^{152}$, C. Stevens ${ }^{135}$, B. Stevenson ${ }^{71}$, T. Stolarczyk ${ }^{4}$, G. Stratta ${ }^{21}$, U. Straumann ${ }^{208}$, J. Strišković ${ }^{166}$, M. Strzys ${ }^{2}$, R. Stuik ${ }^{174}$, M. Suchenek ${ }^{211}$, Y. Suda ${ }^{140}$, Y. Sunada ${ }^{179}$, T. Suomijarvi ${ }^{73}$, T. Suric ${ }^{212}$, P. Sutcliffe ${ }^{153}$, H. Suzuki $^{213}$, P. Świerk ${ }^{101}$, T. Szepieniec ${ }^{152}$, A. Tacchini ${ }^{21}$, K. Tachihara ${ }^{141}$, G. Tagliaferri ${ }^{95}$, H. Tajima ${ }^{139}$, N. Tajima ${ }^{2}$, D. Tak ${ }^{52}$, K. Takahashi ${ }^{214}$, H. Takahashi ${ }^{140}$, M. Takahashi ${ }^{2}$, M. Takahashi ${ }^{2}$, J. Takata $^{2}$, R. Takeishi $^{2}$, T. Tam ${ }^{2}$, M. Tanaka ${ }^{182}$, T. Tanaka ${ }^{213}$, S. Tanaka ${ }^{209}$, D. Tateishi ${ }^{179}$, M. Tavani ${ }^{99}$, F. Tavecchio ${ }^{95}$, T. Tavernier ${ }^{89}$, L. Taylor ${ }^{135}$, A. Taylor ${ }^{52}$, L.A. Tejedor ${ }^{11}$, P. Temnikov ${ }^{189}$, Y. Terada ${ }^{179}$, K. Terauchi ${ }^{180}$, J.C. Terrazas ${ }^{192}$, R. Terrier ${ }^{85}$, T. Terzic ${ }^{121}$, M. Teshima ${ }^{105,2}$, V. Testa ${ }^{28}$, D. Thibaut ${ }^{85}$, F. Thocquenne ${ }^{75}$, W. Tian $^{2}$, L. Tibaldo ${ }^{87}$, A. Tiengo ${ }^{215}$, D. Tiziani ${ }^{142}$, M. Tluczykont ${ }^{50}$, C.J. Todero Peixoto ${ }^{102}$, F. Tokanai ${ }^{154}$, K. Toma ${ }^{160}$, L. Tomankova ${ }^{142}$, J. Tomastik ${ }^{104}$, D. Tonev ${ }^{189}$, M. Tornikoski ${ }^{216}$, D.F. Torres ${ }^{13}$, E. Torresi ${ }^{21}$, G. Tosti ${ }^{95}$, L. Tosti ${ }^{23}$, T. Totani ${ }^{51}$, N. Tothill ${ }^{117}$, F. Toussenel ${ }^{79}$,

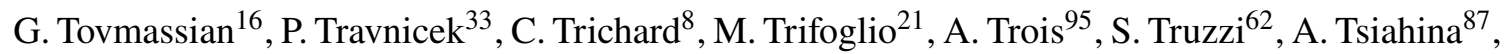

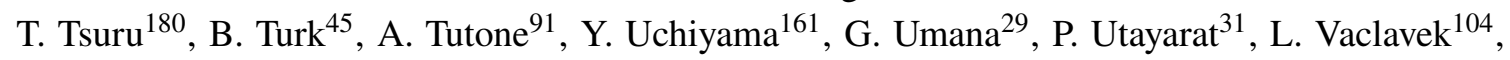
M. Vacula ${ }^{104}$, V. Vagelli ${ }^{23,217}$, F. Vagnetti ${ }^{25}$, F. Vakili $^{218}$, J.A. Valdivia ${ }^{192}$, M. Valentino ${ }^{24}$, A. Valio ${ }^{19}$, B. Vallage ${ }^{89}$, P. Vallania ${ }^{44,64}$, J.V. Valverde Quispe ${ }^{8}$, A.M. Van den Berg ${ }^{42}$, W. van Driel $^{20}$, C. van Eldik $^{142}$, C. van Rensburg ${ }^{1}$, B. van Soelen ${ }^{210}$, J. Vandenbroucke ${ }^{135}$, J. Vanderwalt ${ }^{1}$, G. Vasileiadis ${ }^{39}$, V. Vassiliev ${ }^{71}$, M. Vázquez Acosta ${ }^{32}$, M. Vecchi ${ }^{42}$, A. Vega ${ }^{98}$, J. Veh $^{142}$, P. Veitch $^{118}$, P. Venault ${ }^{75}$, C. Venter ${ }^{1}$, S. Ventura ${ }^{62}$, S. Vercellone ${ }^{95}$, S. Vergani ${ }^{20}$, V. Verguilov ${ }^{189}$, G. Verna $^{27}$, S. Vernetto $^{44,64}$, V. Verzi ${ }^{25}$, G.P. Vettolani ${ }^{90}$, C. Veyssiere ${ }^{144}$, I. Viale ${ }^{55}$, A. Viana ${ }^{80}$, N. Viaux ${ }^{35}$, J. Vicha ${ }^{33}$, J. Vignatti ${ }^{35}$, C.F. Vigorito ${ }^{64,108}$, J. Villanueva ${ }^{98}$, J. Vink ${ }^{174}$, V. Vitale ${ }^{23}$, V. Vittorini ${ }^{99}$, V. Vodeb ${ }^{68}$, H. Voelk ${ }^{53}$, N. Vogel ${ }^{142}$, V. Voisin ${ }^{79}$, S. Vorobiov ${ }^{68}$, I. Vovk ${ }^{2}$, M. Vrastil ${ }^{33}$, T. Vuillaume ${ }^{45}$, S.J. Wagner ${ }^{170}$, R. Wagner ${ }^{105}$, P. Wagner ${ }^{52}$, K. Wakazono ${ }^{139}$, S.P. Wakely ${ }^{127}$, R. Walter ${ }^{38}$, M. Ward ${ }^{5}$, D. Warren ${ }^{54}$, J. Watson ${ }^{52}$, N. Webb ${ }^{87}$, M. Wechakama ${ }^{31}$, P. Wegner $^{52}$, A. Weinstein ${ }^{129}$, C. Weniger ${ }^{174}$, F. Werner ${ }^{53}$, H. Wetteskind ${ }^{105}$, M. White ${ }^{118}$, R. White ${ }^{53}$, A. Wierzcholska ${ }^{101}$, S. Wiesand ${ }^{52}$, R. Wijers ${ }^{174}$, M. Wilkinson ${ }^{124}$, M. Will ${ }^{105}$, D.A. Williams ${ }^{143}$, J. Williams ${ }^{124}$, T. Williamson ${ }^{162}$, A. Wolter ${ }^{95}$, Y.W. Wong ${ }^{142}$, M. Wood ${ }^{96}$, C. Wunderlich ${ }^{62}$, T. Yamamoto ${ }^{213}$, H. Yamamoto ${ }^{141}$, Y. Yamane ${ }^{141}$, R. Yamazaki ${ }^{209}$, S. Yanagita ${ }^{176}$, L. Yang ${ }^{205}$, S. Yoo ${ }^{180}$, T. Yoshida $^{176}$, T. Yoshikoshi $^{2}$, P. $\mathrm{Yu}^{71}$, P. $\mathrm{Yu}^{85}$, A. Yusafzai ${ }^{59}$, M. Zacharias ${ }^{20}$, G. Zaharijas ${ }^{68}$, B. Zaldivar ${ }^{14}$, L. Zampieri ${ }^{76}$, R. Zanmar Sanchez ${ }^{29}$, D. Zaric ${ }^{150}$, M. Zavrtanik ${ }^{68}$, D. Zavrtanik ${ }^{68}$, A.A. Zdziarski ${ }^{49}$, A. Zech ${ }^{20}$, H. Zechlin ${ }^{64}$, A. Zenin ${ }^{139}$, A. Zerwekh ${ }^{35}$, V.I. Zhdanov ${ }^{136}$, K. Ziętara ${ }^{169}$, A. Zink ${ }^{142}$, J. Ziółkowski ${ }^{49}$, V. Zitelli ${ }^{21}$, M. Živec ${ }^{68}$, A. Zmija ${ }^{142}$

1 : Centre for Space Research, North-West University, Potchefstroom, 2520, South Africa

2 : Institute for Cosmic Ray Research, University of Tokyo, 5-1-5, Kashiwa-no-ha, Kashiwa, Chiba 277-8582, Japan

3: Pontificia Universidad Católica de Chile, Av. Libertador Bernardo O’Higgins 340, Santiago, Chile

4 : AIM, CEA, CNRS, Université Paris-Saclay, Université Paris Diderot, Sorbonne Paris Cité, CEA Paris-Saclay, IRFU/DAp, Bat 709, Orme des Merisiers, 91191 Gif-sur-Yvette, France

5 : Centre for Advanced Instrumentation, Dept. of Physics, Durham University, South Road, 
Durham DH1 3LE, United Kingdom

6: Port d'Informació Científica, Edifici D, Carrer de l'Albareda, 08193 Bellaterrra (Cerdanyola del Vallès), Spain

7 : School of Physics and Astronomy, Monash University, Melbourne, Victoria 3800, Australia

8 : Laboratoire Leprince-Ringuet, École Polytechnique (UMR 7638, CNRS/IN2P3, Institut Polytechnique de Paris), 91128 Palaiseau, France

9: Department of Physics, Columbia University, 538 West 120th Street, New York, NY 10027, USA

10 : University of Oslo, Department of Physics, Sem Saelandsvei 24 - PO Box 1048 Blindern, $\mathrm{N}-0316$ Oslo, Norway

11 : EMFTEL department and IPARCOS, Universidad Complutense de Madrid, 28040 Madrid, Spain

12: Instituto de Astrofísica de Andalucía-CSIC, Glorieta de la Astronomía s/n, 18008, Granada, Spain

13 : Institute of Space Sciences (ICE-CSIC), and Institut d'Estudis Espacials de Catalunya (IEEC), and Institució Catalana de Recerca I Estudis Avançats (ICREA), Campus UAB, Carrer de Can Magrans, s/n 08193 Cerdanyola del Vallés, Spain

14 : Instituto de Física Teórica UAM/CSIC and Departamento de Física Teórica, Universidad Autónoma de Madrid, c/ Nicolás Cabrera 13-15, Campus de Cantoblanco UAM, 28049 Madrid, Spain

15 : Dublin Institute for Advanced Studies, 31 Fitzwilliam Place, Dublin 2, Ireland

16 : Universidad Nacional Autónoma de México, Delegación Coyoacán, 04510 Ciudad de México, Mexico

17 : University of Geneva - Département de physique nucléaire et corpusculaire, 24 rue du Général-Dufour, 1211 Genève 4, Switzerland

18 : INFN Dipartimento di Scienze Fisiche e Chimiche - Università degli Studi dell'Aquila and Gran Sasso Science Institute, Via Vetoio 1, Viale Crispi 7, 67100 L'Aquila, Italy

19 : Instituto de Astronomia, Geofísico, e Ciências Atmosféricas - Universidade de São Paulo, Cidade Universitária, R. do Matão, 1226, CEP 05508-090, São Paulo, SP, Brazil

20 : LUTH, GEPI and LERMA, Observatoire de Paris, CNRS, PSL University, 5 place Jules Janssen, 92190, Meudon, France

21 : INAF - Osservatorio di Astrofisica e Scienza dello spazio di Bologna, Via Piero Gobetti 93/3, 40129 Bologna, Italy

22: INAF - Osservatorio Astrofisico di Arcetri, Largo E. Fermi, 5 - 50125 Firenze, Italy

23 : INFN Sezione di Perugia and Università degli Studi di Perugia, Via A. Pascoli, 06123 Perugia, Italy

24 : INFN Sezione di Napoli, Via Cintia, ed. G, 80126 Napoli, Italy

25 : INFN Sezione di Roma Tor Vergata, Via della Ricerca Scientifica 1, 00133 Rome, Italy

26 : Argonne National Laboratory, 9700 S. Cass Avenue, Argonne, IL 60439, USA

27 : Aix-Marseille Université, CNRS/IN2P3, CPPM, 163 Avenue de Luminy, 13288 Marseille cedex 09, France

28 : INAF - Osservatorio Astronomico di Roma, Via di Frascati 33, 00040, Monteporzio Catone, Italy 
29: INAF - Osservatorio Astrofisico di Catania, Via S. Sofia, 78, 95123 Catania, Italy

30 : Grupo de Electronica, Universidad Complutense de Madrid, Av. Complutense s/n, 28040 Madrid, Spain

31: National Astronomical Research Institute of Thailand, 191 Huay Kaew Rd., Suthep, Muang, Chiang Mai, 50200, Thailand

32 : Instituto de Astrofísica de Canarias and Departamento de Astrofísica, Universidad de La Laguna, La Laguna, Tenerife, Spain

33 : FZU - Institute of Physics of the Czech Academy of Sciences, Na Slovance 1999/2, 182 21 Praha 8, Czech Republic

34 : Astronomical Institute of the Czech Academy of Sciences, Bocni II 1401 - 14100 Prague, Czech Republic

35 : CCTVal, Universidad Técnica Federico Santa María, Avenida España 1680, Valparaíso, Chile

36 : ETH Zurich, Institute for Particle Physics, Schafmattstr. 20, CH-8093 Zurich, Switzerland

37 : The University of Manitoba, Dept of Physics and Astronomy, Winnipeg, Manitoba R3T 2N2, Canada

38 : Department of Astronomy, University of Geneva, Chemin d'Ecogia 16, CH-1290 Versoix, Switzerland

39: Laboratoire Univers et Particules de Montpellier, Université de Montpellier, CNRS/IN2P3, CC 72, Place Eugène Bataillon, F-34095 Montpellier Cedex 5, France

40 : Centro Brasileiro de Pesquisas Físicas, Rua Xavier Sigaud 150, RJ 22290-180, Rio de Janeiro, Brazil

41 : Institut de Fisica d'Altes Energies (IFAE), The Barcelona Institute of Science and Technology, Campus UAB, 08193 Bellaterra (Barcelona), Spain

42: University of Groningen, KVI - Center for Advanced Radiation Technology, Zernikelaan 25, 9747 AA Groningen, The Netherlands

43 : School of Physics, University of New South Wales, Sydney NSW 2052, Australia

44 : INAF - Osservatorio Astrofisico di Torino, Strada Osservatorio 20, 10025 Pino Torinese (TO), Italy

45 : Univ. Savoie Mont Blanc, CNRS, Laboratoire d'Annecy de Physique des Particules IN2P3, 74000 Annecy, France

46 : Department of Physics, TU Dortmund University, Otto-Hahn-Str. 4, 44221 Dortmund, Germany

47 : University of Zagreb, Faculty of electrical engineering and computing, Unska 3, 10000 Zagreb, Croatia

48 : University of Namibia, Department of Physics, 340 Mandume Ndemufayo Ave., Pioneerspark, Windhoek, Namibia

49 : Nicolaus Copernicus Astronomical Center, Polish Academy of Sciences, ul. Bartycka 18, 00-716 Warsaw, Poland

50 : Universität Hamburg, Institut für Experimentalphysik, Luruper Chaussee 149, 22761 Hamburg, Germany

51 : Graduate School of Science, University of Tokyo, 7-3-1 Hongo, Bunkyo-ku, Tokyo 113-0033, Japan 
52 : Deutsches Elektronen-Synchrotron, Platanenallee 6, 15738 Zeuthen, Germany

53 : Max-Planck-Institut für Kernphysik, Saupfercheckweg 1, 69117 Heidelberg, Germany

54 : RIKEN, Institute of Physical and Chemical Research, 2-1 Hirosawa, Wako, Saitama, 351-0198, Japan

55 : INFN Sezione di Padova and Università degli Studi di Padova, Via Marzolo 8, 35131 Padova, Italy

56 : Escuela Politécnica Superior de Jaén, Universidad de Jaén, Campus Las Lagunillas s/n, Edif. A3, 23071 Jaén, Spain

57 : Department of Physics and Electrical Engineering, Linnaeus University, 35195 Växjö, Sweden

58 : University of the Witwatersrand, 1 Jan Smuts Avenue, Braamfontein, 2000 Johannesburg, South Africa

59: Institut für Theoretische Physik, Lehrstuhl IV: Plasma-Astroteilchenphysik, Ruhr-Universität Bochum, Universitätsstraße 150, 44801 Bochum, Germany

60 : Faculty of Physics and Applied Computer Science, University of Lódź, ul. Pomorska 149-153, 90-236 Lódź, Poland

61 : INAF - Istituto di Astrofisica Spaziale e Fisica Cosmica di Milano, Via A. Corti 12, 20133 Milano, Italy

62 : INFN and Università degli Studi di Siena, Dipartimento di Scienze Fisiche, della Terra e dell'Ambiente (DSFTA), Sezione di Fisica, Via Roma 56, 53100 Siena, Italy

63 : Center for Astrophysics | Harvard \& Smithsonian, 60 Garden St, Cambridge, MA 02180, USA

64 : INFN Sezione di Torino, Via P. Giuria 1, 10125 Torino, Italy

65 : Finnish Centre for Astronomy with ESO, University of Turku, Finland, FI-20014 University of Turku, Finland

66 : Pidstryhach Institute for Applied Problems in Mechanics and Mathematics NASU, 3B Naukova Street, Lviv, 79060, Ukraine

67 : Bhabha Atomic Research Centre, Trombay, Mumbai 400085, India

68 : Center for Astrophysics and Cosmology, University of Nova Gorica, Vipavska 11c, 5270 Ajdovščina, Slovenia

69 : Institut für Astronomie und Astrophysik, Universität Tübingen, Sand 1, 72076 Tübingen, Germany

70 : Research School of Astronomy and Astrophysics, Australian National University, Canberra ACT 0200, Australia

71 : Department of Physics and Astronomy, University of California, Los Angeles, CA 90095, USA

72 : INFN Sezione di Bari and Politecnico di Bari, via Orabona 4, 70124 Bari, Italy

73 : Laboratoire de Physique des 2 infinis, Irene Joliot-Curie,IN2P3/CNRS, Université ParisSaclay, Université de Paris, 15 rue Georges Clemenceau, 91406 Orsay, Cedex, France

74 : INFN Sezione di Pisa, Largo Pontecorvo 3, 56217 Pisa, Italy

75 : IRFU/DEDIP, CEA, Université Paris-Saclay, Bat 141, 91191 Gif-sur-Yvette, France

76 : INAF - Osservatorio Astronomico di Padova, Vicolo dell'Osservatorio 5, 35122 Padova, Italy 
77 : INAF - Osservatorio Astronomico di Palermo "G.S. Vaiana", Piazza del Parlamento 1, 90134 Palermo, Italy

78 : School of Physics, University of Sydney, Sydney NSW 2006, Australia

79 : Sorbonne Université, Université Paris Diderot, Sorbonne Paris Cité, CNRS/IN2P3, Laboratoire de Physique Nucléaire et de Hautes Energies, LPNHE, 4 Place Jussieu, F-75005 Paris, France

80 : Instituto de Física de São Carlos, Universidade de São Paulo, Av. Trabalhador Sãocarlense, 400 - CEP 13566-590, São Carlos, SP, Brazil

81 : Departament de Física Quàntica i Astrofísica, Institut de Ciències del Cosmos, Universitat de Barcelona, IEEC-UB, Martí i Franquès, 1, 08028, Barcelona, Spain

82 : Department of Physics, Washington University, St. Louis, MO 63130, USA

83 : Saha Institute of Nuclear Physics, Bidhannagar, Kolkata-700 064, India

84 : INAF - Osservatorio Astronomico di Capodimonte, Via Salita Moiariello 16, 80131 Napoli, Italy

85 : Université de Paris, CNRS, Astroparticule et Cosmologie, 10, rue Alice Domon et Léonie Duquet, 75013 Paris Cedex 13, France

86 : Astronomy Department of Faculty of Physics, Sofia University, 5 James Bourchier Str., 1164 Sofia, Bulgaria

87 : Institut de Recherche en Astrophysique et Planétologie, CNRS-INSU, Université Paul Sabatier, 9 avenue Colonel Roche, BP 44346, 31028 Toulouse Cedex 4, France

88 : School of Physics and Astronomy, University of Minnesota, 116 Church Street S.E. Minneapolis, Minnesota 55455-0112, USA

89 : IRFU, CEA, Université Paris-Saclay, Bât 141, 91191 Gif-sur-Yvette, France

90 : INAF - Istituto di Radioastronomia, Via Gobetti 101, 40129 Bologna, Italy

91 : INAF - Istituto di Astrofisica Spaziale e Fisica Cosmica di Palermo, Via U. La Malfa 153, 90146 Palermo, Italy

92 : Astronomical Observatory, Department of Physics, University of Warsaw, Aleje Ujazdowskie 4, 00478 Warsaw, Poland

93: Armagh Observatory and Planetarium, College Hill, Armagh BT61 9DG, United Kingdom

94 : INFN Sezione di Catania, Via S. Sofia 64, 95123 Catania, Italy

95 : INAF - Osservatorio Astronomico di Brera, Via Brera 28, 20121 Milano, Italy

96 : Kavli Institute for Particle Astrophysics and Cosmology, Department of Physics and SLAC National Accelerator Laboratory, Stanford University, 2575 Sand Hill Road, Menlo Park, CA 94025, USA

97 : Universidade Cruzeiro do Sul, Núcleo de Astrofísica Teórica (NAT/UCS), Rua Galvão Bueno 8687, Bloco B, sala 16, Libertade 01506-000 - São Paulo, Brazil

98 : Universidad de Valparaíso, Blanco 951, Valparaiso, Chile

99 : INAF - Istituto di Astrofisica e Planetologia Spaziali (IAPS), Via del Fosso del Cavaliere 100, 00133 Roma, Italy

100 : Lund Observatory, Lund University, Box 43, SE-22100 Lund, Sweden

101 : The Henryk Niewodniczański Institute of Nuclear Physics, Polish Academy of Sciences, ul. Radzikowskiego 152, 31-342 Cracow, Poland 
102 : Escola de Engenharia de Lorena, Universidade de São Paulo, Área I - Estrada Municipal do Campinho, $\mathrm{s} / \mathrm{n}^{\circ}$, CEP 12602-810, Pte. Nova, Lorena, Brazil

103 : INFN Sezione di Trieste and Università degli Studi di Udine, Via delle Scienze 208, 33100 Udine, Italy

104 : Palacky University Olomouc, Faculty of Science, RCPTM, 17. listopadu 1192/12, 771 46 Olomouc, Czech Republic

105 : Max-Planck-Institut für Physik, Föhringer Ring 6, 80805 München, Germany

106 : CENBG, Univ. Bordeaux, CNRS-IN2P3, UMR 5797, 19 Chemin du Solarium, CS 10120, F-33175 Gradignan Cedex, France

107 : Dublin City University, Glasnevin, Dublin 9, Ireland

108 : Dipartimento di Fisica - Universitá degli Studi di Torino, Via Pietro Giuria 1 - 10125 Torino, Italy

109 : Tata Institute of Fundamental Research, Homi Bhabha Road, Colaba, Mumbai 400005, India

110 : Universitá degli Studi di Napoli "Federico II" - Dipartimento di Fisica "E. Pancini", Complesso universitario di Monte Sant'Angelo, Via Cintia - 80126 Napoli, Italy

111 : Oskar Klein Centre, Department of Physics, University of Stockholm, Albanova, SE10691, Sweden

112 : Yale University, Department of Physics and Astronomy, 260 Whitney Avenue, New Haven, CT 06520-8101, USA

113 : CIEMAT, Avda. Complutense 40, 28040 Madrid, Spain

114 : University of Oxford, Department of Physics, Denys Wilkinson Building, Keble Road, Oxford OX1 3RH, United Kingdom

115 : School of Physics \& Astronomy, University of Southampton, University Road, Southampton SO17 1BJ, United Kingdom

116: Department of Physics and Technology, University of Bergen, Museplass 1, 5007 Bergen, Norway

117 : Western Sydney University, Locked Bag 1797, Penrith, NSW 2751, Australia

118 : School of Physical Sciences, University of Adelaide, Adelaide SA 5005, Australia

119 : INFN Sezione di Roma La Sapienza, P.le Aldo Moro, 2 - 00185 Roma, Italy

120 : INFN Sezione di Bari, via Orabona 4, 70126 Bari, Italy

121 : University of Rijeka, Department of Physics, Radmile Matejcic 2, 51000 Rijeka, Croatia

122 : Institute for Theoretical Physics and Astrophysics, Universität Würzburg, Campus Hubland Nord, Emil-Fischer-Str. 31, 97074 Würzburg, Germany

123 : Universidade Federal Do Paraná - Setor Palotina, Departamento de Engenharias e Exatas, Rua Pioneiro, 2153, Jardim Dallas, CEP: 85950-000 Palotina, Paraná, Brazil

124 : Dept. of Physics and Astronomy, University of Leicester, Leicester, LE1 7RH, United Kingdom

125 : Univ. Grenoble Alpes, CNRS, IPAG, 414 rue de la Piscine, Domaine Universitaire, 38041 Grenoble Cedex 9, France

126 : National Centre for nuclear research (Narodowe Centrum Badań Jądrowych), Ul. Andrzeja Sołtana7, 05-400 Otwock, Świerk, Poland 
127 : Enrico Fermi Institute, University of Chicago, 5640 South Ellis Avenue, Chicago, IL 60637, USA

128 : Institut für Physik \& Astronomie, Universität Potsdam, Karl-Liebknecht-Strasse 24/25, 14476 Potsdam, Germany

129 : Department of Physics and Astronomy, Iowa State University, Zaffarano Hall, Ames, IA 50011-3160, USA

130 : School of Physics, Aristotle University, Thessaloniki, 54124 Thessaloniki, Greece

131 : King's College London, Strand, London, WC2R 2LS, United Kingdom

132 : Escola de Artes, Ciências e Humanidades, Universidade de São Paulo, Rua Arlindo Bettio, CEP 03828-000, 1000 São Paulo, Brazil

133 : Dept. of Astronomy \& Astrophysics, Pennsylvania State University, University Park, PA 16802, USA

134 : National Technical University of Athens, Department of Physics, Zografos 9, 15780 Athens, Greece

135 : University of Wisconsin, Madison, 500 Lincoln Drive, Madison, WI, 53706, USA

136 : Astronomical Observatory of Taras Shevchenko National University of Kyiv, 3 Observatorna Street, Kyiv, 04053, Ukraine

137 : Department of Physics, Purdue University, West Lafayette, IN 47907, USA

138: Unitat de Física de les Radiacions, Departament de Física, and CERES-IEEC, Universitat Autònoma de Barcelona, Edifici C3, Campus UAB, 08193 Bellaterra, Spain

139 : Institute for Space-Earth Environmental Research, Nagoya University, Chikusa-ku, Nagoya 464-8601, Japan

140 : Department of Physical Science, Hiroshima University, Higashi-Hiroshima, Hiroshima 739-8526, Japan

141 : Department of Physics, Nagoya University, Chikusa-ku, Nagoya, 464-8602, Japan

142 : Friedrich-Alexander-Universität Erlangen-Nürnberg, Erlangen Centre for Astroparticle Physics (ECAP), Erwin-Rommel-Str. 1, 91058 Erlangen, Germany

143 : Santa Cruz Institute for Particle Physics and Department of Physics, University of California, Santa Cruz, 1156 High Street, Santa Cruz, CA 95064, USA

144 : IRFU / DIS, CEA, Université de Paris-Saclay, Bat 123, 91191 Gif-sur-Yvette, France

145 : INFN Sezione di Trieste and Università degli Studi di Trieste, Via Valerio 2 I, 34127 Trieste, Italy

146: School of Physics \& Center for Relativistic Astrophysics, Georgia Institute of Technology, 837 State Street, Atlanta, Georgia, 30332-0430, USA

147 : Alikhanyan National Science Laboratory, Yerevan Physics Institute, 2 Alikhanyan Brothers St., 0036, Yerevan, Armenia

148 : INAF - Telescopio Nazionale Galileo, Roche de los Muchachos Astronomical Observatory, 38787 Garafia, TF, Italy

149 : INFN Sezione di Bari and Università degli Studi di Bari, via Orabona 4, 70124 Bari, Italy

150 : University of Split - FESB, R. Boskovica 32, 21000 Split, Croatia

151 : Universidad Andres Bello, República 252, Santiago, Chile

152 : Academic Computer Centre CYFRONET AGH, ul. Nawojki 11, 30-950 Cracow, Poland 
153 : University of Liverpool, Oliver Lodge Laboratory, Liverpool L69 7ZE, United Kingdom

154 : Department of Physics, Yamagata University, Yamagata, Yamagata 990-8560, Japan

155 : Astronomy Department, Adler Planetarium and Astronomy Museum, Chicago, IL 60605, USA

156 : Faculty of Management Information, Yamanashi-Gakuin University, Kofu, Yamanashi 400-8575, Japan

157 : Department of Physics, Tokai University, 4-1-1, Kita-Kaname, Hiratsuka, Kanagawa 259-1292, Japan

158 : Centre for Astrophysics Research, Science \& Technology Research Institute, University of Hertfordshire, College Lane, Hertfordshire AL10 9AB, United Kingdom

159 : Cherenkov Telescope Array Observatory, Saupfercheckweg 1, 69117 Heidelberg, Germany

160 : Tohoku University, Astronomical Institute, Aobaku, Sendai 980-8578, Japan

161 : Department of Physics, Rikkyo University, 3-34-1 Nishi-Ikebukuro, Toshima-ku, Tokyo, Japan

162 : Department of Physics and Astronomy and the Bartol Research Institute, University of Delaware, Newark, DE 19716, USA

163 : Institut für Astro- und Teilchenphysik, Leopold-Franzens-Universität, Technikerstr. 25/8, 6020 Innsbruck, Austria

164 : Department of Physics and Astronomy, University of Utah, Salt Lake City, UT 841120830, USA

165 : IMAPP, Radboud University Nijmegen, P.O. Box 9010, 6500 GL Nijmegen, The Netherlands

166: Josip Juraj Strossmayer University of Osijek, Trg Ljudevita Gaja 6, 31000 Osijek, Croatia

167 : Department of Earth and Space Science, Graduate School of Science, Osaka University, Toyonaka 560-0043, Japan

168 : Yukawa Institute for Theoretical Physics, Kyoto University, Kyoto 606-8502, Japan

169 : Astronomical Observatory, Jagiellonian University, ul. Orla 171, 30-244 Cracow, Poland

170 : Landessternwarte, Zentrum für Astronomie der Universität Heidelberg, Königstuhl 12, 69117 Heidelberg, Germany

171 : University of Alabama, Tuscaloosa, Department of Physics and Astronomy, Gallalee Hall, Box 870324 Tuscaloosa, AL 35487-0324, USA

172 : Department of Physics, University of Bath, Claverton Down, Bath BA2 7AY, United Kingdom

173 : University of Iowa, Department of Physics and Astronomy, Van Allen Hall, Iowa City, IA 52242, USA

174 : Anton Pannekoek Institute/GRAPPA, University of Amsterdam, Science Park 9041098 XH Amsterdam, The Netherlands

175 : Faculty of Computer Science, Electronics and Telecommunications, AGH University of Science and Technology, Kraków, al. Mickiewicza 30, 30-059 Cracow, Poland

176 : Faculty of Science, Ibaraki University, Mito, Ibaraki, 310-8512, Japan

177 : Faculty of Science and Engineering, Waseda University, Shinjuku, Tokyo 169-8555, Japan 
178 : Institute of Astronomy, Faculty of Physics, Astronomy and Informatics, Nicolaus Copernicus University in Toruń, ul. Grudziądzka 5, 87-100 Toruń, Poland

179: Graduate School of Science and Engineering, Saitama University, 255 Simo-Ohkubo, Sakura-ku, Saitama city, Saitama 338-8570, Japan

180 : Division of Physics and Astronomy, Graduate School of Science, Kyoto University, Sakyo-ku, Kyoto, 606-8502, Japan

181 : Centre for Quantum Technologies, National University Singapore, Block S15, 3 Science Drive 2, Singapore 117543, Singapore

182 : Institute of Particle and Nuclear Studies, KEK (High Energy Accelerator Research Organization), 1-1 Oho, Tsukuba, 305-0801, Japan

183 : Department of Physics and Astronomy, University of Sheffield, Hounsfield Road, Sheffield S3 7RH, United Kingdom

184 : Centro de Ciências Naturais e Humanas, Universidade Federal do ABC, Av. dos Estados, 5001, CEP: 09.210-580, Santo André - SP, Brazil

185 : Dipartimento di Fisica e Astronomia, Sezione Astrofisica, Universitá di Catania, Via S. Sofia 78, I-95123 Catania, Italy

186 : Department of Physics, Humboldt University Berlin, Newtonstr. 15, 12489 Berlin, Germany

187 : Texas Tech University, 2500 Broadway, Lubbock, Texas 79409-1035, USA

188 : University of Zielona Góra, ul. Licealna 9, 65-417 Zielona Góra, Poland

189 : Institute for Nuclear Research and Nuclear Energy, Bulgarian Academy of Sciences, 72 boul. Tsarigradsko chaussee, 1784 Sofia, Bulgaria

190 : University of Białystok, Faculty of Physics, ul. K. Ciołkowskiego 1L, 15-254 Białystok, Poland

191 : Faculty of Physics, National and Kapodestrian University of Athens, Panepistimiopolis, 15771 Ilissia, Athens, Greece

192 : Universidad de Chile, Av. Libertador Bernardo O’Higgins 1058, Santiago, Chile

193 : Hiroshima Astrophysical Science Center, Hiroshima University, Higashi-Hiroshima, Hiroshima 739-8526, Japan

194 : Department of Applied Physics, University of Miyazaki, 1-1 Gakuen Kibana-dai Nishi, Miyazaki, 889-2192, Japan

195 : School of Allied Health Sciences, Kitasato University, Sagamihara, Kanagawa 228-8555, Japan

196 : Departamento de Astronomía, Universidad de Concepción, Barrio Universitario S/N, Concepción, Chile

197 : Charles University, Institute of Particle \& Nuclear Physics, V Holešovičkách 2, 18000 Prague 8, Czech Republic

198 : Astronomical Observatory of Ivan Franko National University of Lviv, 8 Kyryla i Mephodia Street, Lviv, 79005, Ukraine

199: Kobayashi-Maskawa Institute (KMI) for the Origin of Particles and the Universe, Nagoya University, Chikusa-ku, Nagoya 464-8602, Japan

200 : Graduate School of Technology, Industrial and Social Sciences, Tokushima University, Tokushima 770-8506, Japan 
201 : Space Research Centre, Polish Academy of Sciences, ul. Bartycka 18A, 00-716 Warsaw, Poland

202 : Instituto de Física - Universidade de São Paulo, Rua do Matão Travessa R Nr.187 CEP 05508-090 Cidade Universitária, São Paulo, Brazil

203 : International Institute of Physics at the Federal University of Rio Grande do Norte, Campus Universitário, Lagoa Nova CEP 59078-970 Rio Grande do Norte, Brazil

204 : University College Dublin, Belfield, Dublin 4, Ireland

205 : Centre for Astro-Particle Physics (CAPP) and Department of Physics, University of Johannesburg, PO Box 524, Auckland Park 2006, South Africa

206 : Departamento de Física, Facultad de Ciencias Básicas, Universidad Metropolitana de Ciencias de la Educación, Santiago, Chile

207 : Núcleo de Formação de Professores - Universidade Federal de São Carlos, Rodovia Washington Luís, km 235 CEP 13565-905 - SP-310 São Carlos - São Paulo, Brazil

208 : Physik-Institut, Universität Zürich, Winterthurerstrasse 190, 8057 Zürich, Switzerland

209 : Department of Physical Sciences, Aoyama Gakuin University, Fuchinobe, Sagamihara, Kanagawa, 252-5258, Japan

210 : University of the Free State, Nelson Mandela Avenue, Bloemfontein, 9300, South Africa

211 : Faculty of Electronics and Information, Warsaw University of Technology, ul. Nowowiejska 15/19, 00-665 Warsaw, Poland

212 : Rudjer Boskovic Institute, Bijenicka 54, 10000 Zagreb, Croatia

213 : Department of Physics, Konan University, Kobe, Hyogo, 658-8501, Japan

214 : Kumamoto University, 2-39-1 Kurokami, Kumamoto, 860-8555, Japan

215 : University School for Advanced Studies IUSS Pavia, Palazzo del Broletto, Piazza della Vittoria 15, 27100 Pavia, Italy

216 : Aalto University, Otakaari 1, 00076 Aalto, Finland

217 : Agenzia Spaziale Italiana (ASI), 00133 Roma, Italy

218 : Observatoire de la Cote d'Azur, Boulevard de l'Observatoire CS34229, 06304 Nice

Cedex 4, Franc 